\title{
Dynamics of photoinduced instability in ferroelectric photorefractive crystals caused by space recharging trap waves
}

\author{
A.N. Morozovska, V.V. Obukhovsky, V.V. Lemeshko \\ Taras Shevchenko Kyiv University, Radiophysic Department, 64 Volodymyrs'ka str., 01033 Kyiv, Ukraine. \\ E-mail:vvo@mail.univ.kiev.ua,morozo@iop.kiev.ua.
}

\begin{abstract}
In the paper we theoretically consider the dynamics of the inner field generated by recharging trap waves propagation and spatial-temporal features of the photoinduced light scattering caused by these phenomena in the ferroelectric photorefractive crystals with two donor levels under steady illumination by laser beam. The transverse instability of the interacting light beams plays an important role and both the photovoltaic current from each donor level and the diffusion mechanisms of carrier transfer are taken into account in the proposed theory. For the first time it has been shown that, under the definite relation between the photoionization velocities and anti-collinear Glass vectors of the donor levels, among all possible scenarios of transverse instabilities the boundary circle will be realized in the perfect crystal. Due to this phenomenon the periodical photoinduced light scattering appears in the system. The great attention has been paid to the generalization of the results in the form of master equation for the inner field dynamics and to the description of the optical autowaves generation in ilmenites. All the main theoretical results are in a good agreement with the available experimental data.
\end{abstract}

Keywords: photorefractive crystals, recharging waves, optical autowaves, master equation.

Paper received 08.04.02; accepted for publication 10.12.02.

\section{Introduction}

Ferroelectric photorefractive crystals (PRC) are one of the basic materials for dynamic holography and information processing. But rather often it is impossible to predict the response of the material to laser illumination. For example, various dynamic effects appear in the scattering light even under steady irradiation by laser beam of these materials: periodic pulsation (optical autowaves [1]), non-stationary maxima [2,3], chaotic bursts and optical vortices [4] and so on. In this paper we consider the dynamic halo scattering appeared in $\mathrm{LiTaO}_{3}$, doped with $0.05 \mathrm{wgh} . \%$ of $\mathrm{Cr}$ [5], and the generation of optical autowaves recorded in $\mathrm{LiNbO}_{3}$, doped with 0.02?0.07 wgh. \% of Fe [6]. Such distinctive features of these effects as a rather regular spatial conic structure and impulselike time behavior lead to the appearance of the scattering ring (hallo) on the screen perpendicular to the pump wave direction. This ring spreads out and temporally disappears. The process is periodic in the case of autowave generation.

For the adequate description of such effects in PRC, it is necessary to evolve the theory in which the transverse instability of the interacting light beams plays an important role and both the photovoltaic (PV) and the diffusion mechanisms of carrier transfer are taken into account [7-11].

We used the following well-known phenomenon as the basic idea for the evolving of the theory. The nonuniform time-dependent inner photoinduced field appears in PRC even under steady laser illumination. It can be detected due to the linear electro-optic (EO) effect. The theoretical interpretation of this phenomenon based on the analysis of space and temporal dependences of the degree of completion of the crystal impurity levels (traps). Under definite conditions, the non-uniform and non-stationary traps completion proved to be energetically preferable. In this case, either damping or periodic recharging trap waves can propagate in the crystal in the linear approximation [12]. The spatial structure and evolution of the scattered light is determined by this inner field in accordance with EO-effect.

In the paper, we consider the dynamics of the inner field generated by recharging trap waves propagation and predict spatial-temporal features of the photoinduced light scattering (PILS) caused by these phenomena. To run ahead, notice that the aforementioned dynamic effects disappear in the theoretical consideration of the model systems with one donor level and traditional PV- 


\section{A.N. Morozovska et al.: Dynamics of photoinduced instability in ferroelectric...}

current density (see for details (15b), where real roots are absent at $\left.\Lambda_{p k}=0\right)$. Thus, as distinct from [12,13] and our previous model [21] with one donor level, but rather specific expression for PV-current density, in this paper we consider the system with two donor levels and we have taken into account that each level supplies photoelectrons to the conductive band, i.e. these two components of the photocurrent exist with different Glass vectors. In contract to [12], we have investigated not only the longitudinal structure of the recharging waves, which exists even in the case of pure longitudinal photovoltaic current, but have paid sufficient attention to their transverse structure appearing due to the transverse photovoltaic current in ilmenites. The authors of [12] have obtained the dispersion relation "frequency - wave vector" for onedimensional waves (i.e. in pure longitudinal case). Below we will derive the analogous relation for three-dimensional waves. In such case, the diffusion current compensates the transverse photovoltaic one owing to the absence of the inner field transverse components. The compensation can lead to the generation and stabilization of the transverse periodical recharging waves. It is possible under the definite frequency and wave vector values depending only on PRC material parameters and laser pump wave intensity. The latter result means that this phenomenon is theoretically possible under the steady illumination of the sample by incoherent but rather powerful light beam. Thus, similar phenomena can be registered under the PILS investigation of a weak coherent signal beam in the crystal irradiated by a powerful incoherent reference light beam with an appropriate wavelength.

\section{The problem}

Let us consider the dynamic photoinduced effects in PRC without inversion center. We will be interested in realization of the transverse instability generation threshold for the electric fields in PRC under steady illumination by a laser beam along polar (optical) axis.

In order to obtain relevant results, let us calculate the interaction of the laser beam with the dynamic hologram (photoinduced spatially non-uniform inner field) recorded by itself in the crystal within the framework of the perturbation theory.

Our calculations correspond to the following geometry: the pump wave vector is directed along the crystal optical axes $\mathrm{Z}$, the pump polarization vector $\overrightarrow{\mathrm{e}}_{p}$ lies in the XY-plane, the coordinate origin coincides with the pump beam center on the front plane of the crystal. The crystal of the $\mathrm{C}_{3 \mathrm{~V}}$ symmetry group is considered infinite in the transverse directions $\mathrm{X}, \mathrm{Y}$ as the pump beam diameter used in the wave equation is much smaller than the transverse crystal sizes, and has definite thickness $l$ in Zdirection.

For this case, we shall find the inner electric field in the non-depleted pump wave approximation with definite intensity. The dynamics of inner field is determined from the system of equations for the degree of completion of donor and acceptor levels by electrons, the concentration of electrons in the conduction band, and from the Maxwell equations, which can be reduced to the Poisson equation for the quasi-static electric field. Hereinafter acceptor levels are considered rather deep and therefore completely filled by electrons, and donor levels are considered to be so shallow, that thermal release of electrons into conduction band is possible.

Then we shall discuss the interaction of the high-frequency laser pump beam with the dynamic holographic grating (recorded by the aforementioned spatial-non-homogeneous photoinduced inner field allowing for linear EO-effect) in the crystal and analyze the features of the diffracted light.

\section{The dynamics of inner field in the constant pump wave approximation}

\subsection{The general system of equations}

In accordance with papers [13] the system of equations for the inner field in the quasi-homogeneous region of the PRC has the form:

$\frac{\partial n_{p}^{+}}{\partial t}=-\gamma_{p} n_{e} n_{p}^{+}+\left(s_{p} I_{0}+s_{0}\right)\left(n_{p}^{0}-n_{p}^{+}\right), \quad p=1,2$,

$\frac{\partial n_{e}}{\partial t}=\frac{\partial n_{1}^{+}}{\partial t}+\frac{\partial n_{2}^{+}}{\partial t}+\frac{d i v \vec{j}_{n}}{e}$

$\operatorname{div}\left(\varepsilon \vec{E}_{f}\right)=4 \pi e\left(-n_{e}+n_{1}^{+}+n_{2}^{+}\right)$

$\vec{j}_{n}=e \mu n_{e} \vec{E}_{f}+e \operatorname{Dgrad}\left(n_{e}\right)+\sum_{p=1,2}\left(n_{p}^{0}-n_{p}^{+}\right) s_{p} I_{0} \vec{G}_{p}$

Where the following designations are introduced: $n_{e}$ is the concentration of free electrons, $n_{p}^{0}$ is the concentration of donors for " $p$ "-level, $n_{p}^{+}$is the concentration of ionized donors (traps) for " $p$ "-level, $\mathrm{g}_{\mathrm{p}}$ is the capture coefficient of electron by the " $p$ "-kind trap, $s_{p}$ is the photoionization coefficients, $I_{0}$ is the pump intensity, $s_{0}$ is the thermalization coefficient, $e$ is the absolute value of the electron charge, $j_{n}$ is the density of electron current , $\varepsilon$ is static dielectric permittivity, $m$ is the electron mobility, $D$ is the diffusion coefficient, $G_{p k}=\beta^{p}{ }_{k l m} e_{l} e_{m}^{*}$ is the Glass vectors (the double convolution of the PVtensor with the pump wave polarization $e_{p}$ ). As distinct from $[12,13]$, in the density of the PV-current (see 1d), we have taken into account that each level supplies photoelectrons to the conductive band, i.e. these two components of the photocurrent exist with different Glass vectors.

The system (1) must be supplemented by initial and boundary conditions. The latter conditions represent the 
field state at the sample boundaries $z=0$ and $z=l$. Let us discuss the cases of free and short-circuited samples. In the literature [14-15], one can find the detailed description of the spontaneous polarization $\left(P_{S}\right)$ screening in the uniaxial ferroelectrics without illumination. The main results of these works can be formulated as the following statements. The screening is internal for the stationary state of single-domain ferroelectric with not rather broad band gap and therefore does not noticeably influence on the distribution of the inner field and spontaneous induction in the sample. Thus, in the case of the short-circuit condition, the sample can be treated as the sample with free surface, and one can apply to it the solutions for the fields obtained in [15] for the free one. These solutions correspond to the small purely longitudinal constant inner field in the bulk of the crystal, i.e. at $\ell_{c}<z<\ell-\ell_{c}$ ( $l$ is the crystal thickness, $l_{c}$ is the correlation length), and sharply dependent over $z$ coordinate strong longitudinal field in the surface layer (SL), i.e. at $0<z<\ell_{c}$, $\ell-\ell_{c}<z<\ell$. Here we would like to emphasize that the influence of the bulk photovoltaic effect is negligibly small in SL because of the negligibly small $P_{s}$ and strong symmetry disturbance near the surface (in [14 p.167], it was shown that $G \sim P_{S}$ ) and thus in this region.

The system (1) has been solved when using the perturbation theory [12] at $\ell_{c}<<\ell$ and the aforementioned statements about the constant inner field.

1) The only stationary (s) spatial uniform solution of (1) can be chosen as zero approximation: $\vec{E}_{s}=\operatorname{const}\left(I_{0}\right) \cdot \vec{G}$ $n_{p s}^{+}=$const, $n_{e s}=$ const , which at $\ell_{c}<z<\ell-\ell_{c}$ is valid either for short-circuited or for free sample. It smoothly sews together with solution [14-15] in the SL due to $\vec{G} \equiv 0$

2) Small dynamic corrections to this stationary solution of (1) can be obtained as the first approximation: $\vec{E}_{f}=\vec{E}_{s}+\widetilde{\vec{E}}_{f}(x, y, z, t), \quad n_{p}^{+}=n_{p s}^{+}+\tilde{n}_{p}^{+}, \quad n_{e}=n_{e s}+\tilde{n}$. In order to use the aforementioned stationary solution [15] as good zero approximation, in accordance with the stability theory, the transverse dynamic components of the inner field must satisfy the trivial boundary conditions in SL: $\tilde{E}_{f_{x, y}}\left(x, y, 0<z<\ell_{c}, t\right)=\tilde{E}_{f_{x, y}}\left(x, y, \ell-\ell_{c}<z<\ell, t\right)=0$. Only these conditions provide the longitudinal form of the field in SL and continuity of its tangential components at the crystal boundaries. If the SL has the real thickness and the solutions are analytic, $\widetilde{E}_{f}(x, y, z, t)=0$. Also the longitudinal dynamic inner field components have to satisfy trivial boundary conditions. They are zero at the internal boundaries of SL for free sample, i.e. $\tilde{E}_{f_{z}}\left(x, y, z=\ell_{c}, t\right)=\tilde{E}_{f}\left(x, y, z=\ell-\ell_{c}, t\right)=0$. In this case, the bulk sources (photoinduced fluctuations in the vicinity of defects) are seeding for the generation of the transverse. The potential difference between the electrodes is zero for 1 short-circuited sample, i.e. $\int_{0}^{\ell} \tilde{E}_{f_{z}}(x, y, z, t) d z=0$, and the photoinduced fluctuations of the inner field at the initial moment $t=0$ can be treated as the seeding.

\subsection{The stationary solution of the system}

Neglecting the existence of circular currents in PRC, in stationary case $(1 \mathrm{~b})$ is reduced to the condition of neglecting the magnetic field in PRC $[9,12]: j_{n}=0$. When taking into account that all values are time independent, one nonlinear equation in particular derivatives for the concentration of free carriers $n_{s}$ can be obtained instead of (1). Its only spatial uniform solution can be represented in the form:

$$
\begin{aligned}
& n_{p s}^{+}=\frac{s_{p} I_{0}+s_{0}}{\gamma_{p} n_{e s}+s_{p} I_{0}+s_{0}} n_{p}^{0}, \\
& \vec{E}_{s}=\sum_{p=1,2} \frac{\gamma_{p} n_{p s}^{+} s_{p} I_{0} \vec{G}_{p}}{e \mu\left(s_{p} I_{0}+s_{0}\right)},
\end{aligned}
$$

where $n_{e s}$ is the root of the following cubic equation:

$$
n_{e s}=\sum_{p=1,2} \frac{s_{p} I_{0}+s_{0}}{\gamma_{p} n_{e s}+s_{p} I_{0}+s_{0}} n_{p}^{0} .
$$

For rather high intensities the expressions $(2,3)$ admit the asymptotic form:

$$
\begin{aligned}
& n_{p s}^{+} \approx \frac{s_{p} I_{0}}{\gamma_{p} n_{e s}+s_{p} I_{0}} n_{p}^{0} \approx \\
& \approx \frac{s_{p} I_{0}}{\gamma_{p}\left(n_{1}^{0}+n_{2}^{0}\right)+s_{p} I_{0}} n_{p}^{0} \rightarrow n_{p}^{0}
\end{aligned}
$$$$
n_{e s} \approx \sum_{p=1,2} \frac{s_{p} I_{0}}{\gamma_{p} n_{e s}+s_{p} I_{0}} n_{p}^{0} \approx
$$

$\approx \sum_{p=1,2} \frac{s_{p} I_{0}}{\gamma_{p}\left(n_{1}^{0}+n_{2}^{0}\right)+s_{p} I_{0}} n_{p}^{0} \rightarrow n_{1}^{0}+n_{2}^{0}$.

Taking into consideration the discussion from item 2.1, we will use (2-4) as zero approximation for inner field dynamics in the bulk of the crystal.

\subsection{Linearized system for dynamic components}

Using (1a, 1c, 1d), after elementary transformations (1b) can be rewritten as:

$$
\begin{aligned}
& \operatorname{div}\left(\frac{\varepsilon}{4 \pi} \frac{\partial}{\partial t} \vec{E}_{f}+e \mu n_{e} \vec{E}_{f}+e \operatorname{Dgrad}\left(n_{e}\right)+\right. \\
& \left.+\sum_{p=1,2}\left(n_{p}^{0}-n_{p}^{+}\right) s_{p} I_{0} \vec{G}_{p}\right)=0 .
\end{aligned}
$$




\section{A.N. Morozovska et al.: Dynamics of photoinduced instability in ferroelectric...}

After neglecting the existence of magnetic field in PRC $[9,12]$, one can obtain that:

$$
\begin{aligned}
& \frac{\varepsilon}{4 \pi} \frac{\partial}{\partial t} \vec{E}_{f}+e \mu n_{e} \vec{E}_{f}+e \operatorname{Dgrad}\left(n_{e}\right)+ \\
& +\sum_{p=1,2}\left(n_{p}^{0}-n_{p}^{+}\right) s_{p} I_{0} \vec{G}_{p}=0 .
\end{aligned}
$$

We will find the solution of (1) in the form:

$$
\begin{array}{lll}
\vec{E}_{f}=\vec{E}_{s}+\tilde{\vec{E}}_{f}(x, y, z, t), & n_{p}^{+}=n_{p s}^{+}+\tilde{n}_{p}^{+}, & n_{e}=n_{e s}+\tilde{n}, \\
\left|\overrightarrow{\vec{E}}_{f}(x, y, z, t)\right|<<\left|\vec{E}_{s}\right|, & \left|\tilde{n}_{p}^{+}\right|<<n_{p s}^{+}, & |\tilde{n}|<<n_{e s},
\end{array}
$$

with the boundary conditions for the inner field in the short-circuited sample:

$$
\begin{aligned}
& E_{f_{x, y}}(x, y, z, t)=0, \quad \int_{0}^{\ell} \tilde{E}_{f_{z}}(x, y, z, t) d z=0, \\
& \tilde{E}_{f_{z}}(x, y, z, t=0)=v(x, y, z), \quad \int_{0}^{\ell} v(x, y, z) d z=0 .
\end{aligned}
$$

Here $v(x, y, z) \div \hat{v}(\vec{q}) \sim I_{0}$ at $\left(\ell_{c}<z<\ell-\ell_{c}\right)$ is small photoinduced fluctuation seeding with rather broad spatial-temporal spectrum. Representation (7) allows us to linearize the system for small dynamic components. In the first approximation the properties of the solutions of (1) should be agreed with the stability theory: at the stability threshold the dynamic components have to be not increasing functions of time $t$ and transverse coordinates $\mathrm{x}, \mathrm{y}$ and analytical functions of $z$, i.e. the Fourier image at variables $t, x, y$ and the Laplace image at variable $z$ must exist. Then:

$$
\begin{aligned}
& \tilde{\vec{E}}_{f}(x, y, z, t)=\int d \vec{q} d \Omega \cdot \vec{U}(\vec{q}, \Omega) \exp (i \vec{q} \vec{r}+i \Omega t), \\
& \tilde{n}(x, y, z, t)=\int d \vec{q} d \Omega \hat{n}(\vec{q}, \Omega) \exp (i \vec{q} \vec{r}+i \Omega t), \\
& \tilde{n}_{p}^{+}(x, y, z, t)=\int d \vec{q} d \Omega \cdot \hat{n}_{p}^{+}(\vec{q}, \Omega) \exp (i \vec{q} \vec{r}+i \Omega t),
\end{aligned}
$$

where $\vec{q} \vec{r}=q_{z} z+q_{x} x+q_{y} y, q_{z}=q_{z}^{\prime}+i q_{z}^{\prime \prime}$,

$$
\left(q_{z}^{\prime}, q_{z}^{\prime \prime}, q_{x}, q_{y}, \Omega\right) \in(-\infty, \infty) \text {. }
$$

Linearized system for images represents itself the homogeneous one of algebraic equations:

$$
\begin{aligned}
& i \Omega \widehat{n}_{p}^{+}=-\gamma_{p} \hat{n} n_{p s}^{+}-\left(s_{p} I_{0}+s_{0}+\gamma_{p} n_{e s}\right) \hat{n}_{p}^{+}, \\
& i(\vec{q} \vec{U})=\frac{4 \pi e}{\varepsilon}\left(\hat{n}_{1}^{+}+\hat{n}_{2}^{+}-\hat{n}\right), \\
& \frac{\varepsilon}{4 \pi} i \Omega \vec{U}+e \mu\left(n_{e s} \vec{U}+\widehat{n} \vec{E}\right)_{s}+i e D \vec{q} \hat{n}-\sum_{p=1,2} \hat{n}_{p}^{+} s_{p} I_{0} \vec{G}_{p}=0 .
\end{aligned}
$$

On solving (10) one can obtain the relations:

$$
\begin{aligned}
& \widehat{n}_{p}^{+}=-\frac{\gamma_{p} n_{p s}^{+}}{i \Omega+s_{p} I_{0}+s_{0}+\gamma_{p} n_{e s}} \widehat{n}, \\
& \widehat{n}=-\frac{i \varepsilon(\vec{q} \vec{U})}{4 \pi e}\left(1+\sum_{p=1,2} \frac{\gamma_{p} n_{p s}^{+}}{i \Omega+s_{p} I_{0}+s_{0}+\gamma_{p} n_{e s}}\right)^{-1},
\end{aligned}
$$

and the vector equation:

$\vec{U}\left(1+i \Omega_{p} m_{p}\right)-i(\vec{Q} \vec{U})\left(1+\sum_{p=1,2} \frac{h_{p}\left(f_{p}-1\right)}{i \Omega_{p}+f_{p}}\right)^{-1} \times$

$$
\times\left(i \vec{Q}-\sum_{p=1,2} \vec{\lambda}_{p}\left(1-\frac{1}{i \Omega_{p}+f_{p}}\right)\right)=0
$$

Before solving the latter vector equation let us rewrite it in appropriate variables in the case when the dark conductivity can be neglected, i.e. $s_{p} I_{0}>>s_{0}$. After this the (11b) has the form:

$$
\begin{aligned}
& \vec{U}\left(1+i \Omega_{p} m_{p}\right)-i(\vec{Q} \vec{U})\left(1+\sum_{p=1,2} \frac{h_{p}\left(f_{p}-1\right)}{i \Omega_{p}+f_{p}}\right)^{-1} \times \\
& \times\left(i \vec{Q}-\sum_{p=1,2} \vec{\lambda}_{p}\left(1-\frac{1}{i \Omega_{p}+f_{p}}\right)\right)=0 .
\end{aligned}
$$

Hereinafter the following designations are used: $\tau_{m}=\varepsilon / 4 \pi e \mu n_{e s}$ is the Maxwellian time, $\tau_{p}=1 / s_{p} I_{0}$ is the photoionization time of electron for "p"-level donors, $m_{p}=\tau_{m} / \tau_{p}$ is the ratio of these times, $S_{p k}=s_{p} / s_{k}$ is the ratio of the photoionization coefficients, $\ell_{d}=\sqrt{\varepsilon D / 4 \pi e \mu n_{e s}}$ is the diffusion length (if the Einstein ratio is valid $e D=\mu k_{B} T$, the diffusion length coincides with the Debye screening radius [14]), $\vec{\ell}_{g p}=\varepsilon \gamma_{p} n_{p s}^{+} \vec{G}_{p} / 4 \pi e^{2} \mu n_{e s}$ is the photovoltaic (drift) length, $\lambda_{p}=\vec{\ell}_{g p} / \ell_{d}$ is the ratio of these lengths, $f_{p}=n_{p}^{0} / n_{p s}^{+}$is the ratio of the donor concentration to the trap one for " $\mathrm{p}$ "-level $\left(f_{p}>1\right), h_{p}=n_{p s}^{+} / n_{e s}$, subscripts $p, k=1,2$. Also the dimensionless variables are introduced: $\vec{Q}=\vec{q} \ell_{d}, \quad \Omega_{p}=\Omega \tau_{p}$.

The expression (12) allowing for the boundary condition (8) $\vec{U}_{\perp}=0$, leads to the dispersion relation for the longitudinal $Q_{z}$ and transverse $Q_{\perp}$ components of the wave vector:

$$
\begin{aligned}
& \left(1+i \Omega_{p} m_{p}\right)-i Q_{z}\left(1+\sum_{p=1,2} \frac{h_{p}\left(f_{p}-1\right)}{i \Omega_{p}+f_{p}}\right)^{-1} \times \\
& \times\left(i Q_{z}-\sum_{p=1,2} \lambda_{p z}\left(1-\frac{1}{i \Omega_{p}+f_{p}}\right)\right)=0
\end{aligned}
$$


$Q_{\perp}=-i \sum_{p=1,2} \lambda_{p \perp}\left(1-\frac{1}{i \Omega_{p}+f_{p}}\right)$

The absolute transverse instability threshold can be determined from the conditions:

$\operatorname{Im}(\Omega)=\operatorname{Im}\left(Q_{\perp}\right)=0$.

Really, the component $Q_{\perp}$ in (14) must be real as the component of the Fourier transform wave vector (9). Thus, one can find the expression for $\mathrm{W}_{\mathrm{f}}$ from the condition $\operatorname{Im}\left(Q_{\perp}\right)=0$ (hereinafter the subscript $\mathrm{f}$ underlines the definite solution of the obtained equations). As the result the more compact expression for $Q_{f \perp}$ can be derived:

$Q_{f \perp}=\sum_{p=1,2} \lambda_{p \perp} \frac{\Omega_{p}}{\Omega_{p}^{2}+f_{p}^{2}}$. form:

The equation for the frequency $\Omega_{p}=\Omega_{f} \tau_{p}$ acquires the

$$
1-\frac{f_{k}}{\Omega_{k}^{2}+f_{k}^{2}}=\Lambda_{p k}\left(1-\frac{f_{p}}{\Omega_{p}^{2}+f_{p}^{2}}\right),
$$

and it is biquadrate. Notice, that real roots are absent in (15b) at $\Lambda_{p k}=0$, therefore the aforementioned dynamic effects disappear in the theoretical consideration of the model systems with one donor level and traditional PVcurrent density.

The expression for the longitudinal wave vector $Q_{z}$ was found from (13) after substitution of $\Omega_{p}$ from (15b). At rather high intensities (see below (19-20) $f_{p} \rightarrow 1$ ) these solutions can be simplified as:

$$
\begin{aligned}
& Q_{f z 1,2} \approx-\sum_{p=1,2} \frac{i \lambda_{p z}}{2}\left(1-\frac{1}{i \Omega_{p}+f_{p}}\right) \mp \\
& \mp \sqrt{\left[\sum_{p=1,2} \frac{i \lambda_{p z}}{2}\left(1-\frac{1}{i \Omega_{p}+f_{p}}\right)\right]^{2}-\left(1+i \Omega_{p} m_{p}\right) .}
\end{aligned}
$$

If the following inequality is valid $\left|\lambda_{p z}\right|>>1$, i.e. the diffusion is small $l_{g}>>l_{d}$, the equation (13) is simplified and the expressions for $Q_{f z}$ obtains the form:

$$
\begin{aligned}
& Q_{f z 1} \approx-i \sum_{p=1,2} \lambda_{p z}\left(1-\frac{1}{i \Omega_{p}+f_{p}}\right)+ \\
& +\frac{\left(1+i \Omega_{p} m_{p}\right)}{\sum_{p=1,2} \lambda_{p z}\left(1-\frac{1}{i \Omega_{p}+f_{p}}\right)}, \\
& Q_{f z 2} \approx-\frac{\left(1+i \Omega_{p} m_{p}\right)}{\sum_{p=1,2} \lambda_{p z}\left(1-\frac{1}{i \Omega_{p}+f_{p}}\right)} .
\end{aligned}
$$

In a general case, we designate the roots of the square equation (13) as $Q_{f z 1}$ and $Q_{f z 2}$.

Let us analyze the existence and find the simplified, suitable for analysis expressions for the roots of (15b). The graphical study of (15b) shows that the sufficient condition of the existence of the only pair of its symmetrical roots is as follows:

$-1<\Lambda_{p k}<-\frac{f_{p}\left(f_{k}-1\right)}{f_{k}\left(f_{p}-1\right)}$.

The more important sequence of (17) is the following statement. The transverse components of Glass vectors for two donor (impurity) levels must be antiparallel. Note, that their co-linearity follows from the assumption that the small photoactive impurity concentration does not break the initial crystal symmetry. The latter determines the structure of photovoltaic tensor, i.e. its non-zero components and the relations between them. Actually, the symmetry of the crystal matrix determines non-zero, independent components of the PV-tensor in the eigen coordinate system, i.e. the existence and the direction of the straight line, the Glass vector is directed along or against it. The magnitude of the Glass vector cannot be determined from the symmetry properties. The donor atoms can generate photoelectrons with different velocities both in the abovementioned direction and in the opposite one depending on their specific properties. What the physical meaning concerning to the origin and structure of these levels can be derived from the anti-colinearity of their Glass vectors components? Up to date we can propose the following model.

Both or at least one of the donor levels is made of photoactive impurity atoms. Firstly, they substitute some kind of lattice atoms or locate in the interstice and join into the first donor level. If the impurity atom valence does not coincide with the substituent atom one, the excess charge appears in lattice, which is not energetically favorable. As a result, at the distances less than a few lattice constants from the impurity atom, the appearance of the compensative charged center, consisted of lattice atoms or another impurity atom in opposite charge state, is a most probable. As a whole, this spatial complex looks like some cluster with a dipole moment and, as a consequence, with some asymmetric potential. The magnitude of the asymmetry is quite enough to cause the noticeable PV-current in the sample [22]. We assume that the aforementioned compensative centers or their nearest neighbors join into the second donor level. The photoelectron generation takes place at the both ends of such complexes. Owing to the different neighbors and opposite charge state of complex atoms, their photoionization and capture coefficients, other parameters are different, and the generated photoelectrons have oppositely directed velocities. For example, the aforementioned compensative centers can rather strongly asymmetrically absorb free carriers. This absorption causes the macroscopic PVcurrent in the non-equilibrium conditions [14, p.174], 


\section{A.N. Morozovska et al.: Dynamics of photoinduced instability in ferroelectric...}

direction of which is opposite to that, generated by the photoionization of the impurity atoms.

Hereinafter we assume that such characteristics of donor level atoms as the photoionization or/and capture coefficient are significantly different (for example, $S_{21}>>1$ ). The similar "nonequivalence" of the levels can significantly simplify the consideration of the problem. Thus, in the case when the inequality is valid:

$$
\left(\frac{S_{21} \Omega_{1}}{f_{2}}\right)^{2}>>1
$$

equation (15b) can be approximately rewritten in the form $1-\frac{f_{1}}{\Omega_{1}^{2}+f_{1}^{2}} \approx \Lambda_{21}$, which has the simple solution

$\Omega_{f} \approx \pm \frac{f_{1}}{\tau_{1}} \sqrt{-\frac{\Lambda_{21}+1-1 / f_{1}}{\Lambda_{21}+1}}$

The latter exists at

$-1<\Lambda_{21}<-1+\frac{1}{f_{1}}$.

Let us study the dependence of the frequency $\mathrm{W}_{\mathrm{f}}$ and wave vector $q_{f}$ from $(15 \mathrm{a}, 18 \mathrm{~b})$ over such material parameters as pump intensity $I_{0}$ and photoactive impurity concentration $n_{d}$.

For the sufficient intensities above the threshold the asymptotic formulae (4) are valid, i.e.:

$$
\begin{aligned}
& f_{p}=\frac{\gamma_{p} n_{e s}}{s_{p} I_{0}}+1 \approx 1+\frac{\gamma_{p}\left(n_{1}^{0}+n_{2}^{0}\right)}{s_{p} I_{0}} \rightarrow 1, \\
& \Lambda_{p k}=\frac{G_{p \perp} n_{p s}^{+}}{G_{k \perp} n_{k s}^{+}} \approx\left(\frac{G_{p \perp} \gamma_{p} s_{p} n_{p}^{0}}{G_{k \perp} \gamma_{k} s_{k} n_{k}^{0}}\right) \frac{\gamma_{k}\left(n_{1}^{0}+n_{2}^{0}\right)+s_{k} I_{0}}{\gamma_{p}\left(n_{1}^{0}+n_{2}^{0}\right)+s_{p} I_{0}} \rightarrow \\
& \rightarrow \frac{G_{p \perp} \gamma_{p} n_{p}^{0}}{G_{k \perp} \gamma_{k} n_{k}^{0}}, \\
& \vec{\ell}_{g p} / \ell_{d}^{2} \approx\left(\frac{\vec{G}_{p} \gamma_{p} n_{p}^{0}}{e D}\right) \frac{s_{p} I_{0}}{\gamma_{p}\left(n_{1}^{0}+n_{2}^{0}\right)+s_{p} I_{0}} \rightarrow \frac{\vec{G}_{p} \gamma_{p} n_{p}^{0}}{e D} .
\end{aligned}
$$

The threshold intensity $I_{C}$ can be determined as the one, at which the real roots of (15b) appear. The more detailed analysis shows that the approximate estimation for $I_{C}$ is valid:

$$
I_{C}=\max _{\substack{k=1,2 \\ p \neq k}}\left\{\frac{\gamma_{k}\left(n_{1}^{0}+n_{2}^{0}\right)}{s_{k}}\left(-\frac{1}{\Lambda_{p k}}-1\right)\right\}
$$

Hereinafter let us suppose that both donor levels were created due to the interstitial of the same impurity atoms to the crystal, then: $n_{p}^{0} \sim n_{d}$.

When substituting of (19) in (15a) and (18b) the following approximate formulae can be obtained:

$$
\begin{aligned}
& q_{f z 1}\left(\xi, N_{d}\right) \approx \sigma q_{f \perp}\left(\xi, N_{d}\right)-\frac{1}{\sigma q_{f \perp}\left(\xi, N_{d}\right)}-i \frac{\Omega_{f}\left(\xi, N_{d}\right) \tau_{m o}}{\sigma q_{f \perp}\left(\xi, N_{d}\right) N_{d}}, \\
& q_{f z 2}\left(\xi, N_{d}\right) \approx \frac{1}{\sigma q_{f \perp}\left(\xi, N_{d}\right)}+i \frac{\Omega_{f}\left(\xi, N_{d}\right) \tau_{m o}}{\sigma q_{f \perp}\left(\xi, N_{d}\right) N_{d}}, \\
& -q_{f z 1}^{\prime \prime}\left(\xi, N_{d}\right)=q_{f z 2}^{\prime \prime}\left(\xi, N_{d}\right) \approx \frac{\Omega_{0} \tau_{m o}}{\sigma q_{0 \perp}\left(\Lambda_{21}+1\right) N_{d}} \frac{\left(\xi / N_{d}+1\right)^{2}}{\xi / N_{d}} .
\end{aligned}
$$

where the dimensionless variables and designations are introduced:

$\xi=s_{1} I_{0} / \gamma_{1} n_{0}, \quad N_{d}=\left(n_{1}^{0}+n_{2}^{0}\right) / n_{0}, \Omega_{0}=\gamma_{1} n_{0}$,

$\vec{q}_{0 \perp}=\frac{\vec{G}_{1 \perp} \gamma_{1} n_{0}}{e D} \frac{n_{1}^{0}}{n_{1}^{0}+n_{2}^{0}}, \quad \sigma=\frac{G_{1 z}}{G_{1 \perp}}=\frac{G_{2 z}}{G_{2 \perp}}$,

$\tau_{m o}=\frac{\varepsilon}{4 \pi e \mu n_{0}}$

$n_{0}$ is the matrix atoms concentration (that is why $N_{d}$ can be measured in percents). It can be easily shown that always $q_{f z 2}^{\prime \prime}=-q_{f z 1}^{\prime \prime}$ and in the case of weak diffusion the inequality $\left|q_{f z 2}^{\prime}\right|<<\left|q_{f z 1}^{\prime}\right|$ is valid. Really, if the inequality $\left|\sigma q_{f \perp}\left(\xi, N_{d}\right)\right|>>1$ is valid, one can derive the approximate expressions:

$$
\begin{aligned}
& q_{f z 1}\left(\xi, N_{d}\right) \approx \sigma q_{f \perp}\left(\xi, N_{d}\right)-\frac{1}{\sigma q_{f \perp}\left(\xi, N_{d}\right)}-i \frac{\Omega_{f}\left(\xi, N_{d}\right) \tau_{m o}}{\sigma q_{f \perp}\left(\xi, N_{d}\right) N_{d}}, \\
& q_{f z 2}\left(\xi, N_{d}\right) \approx \frac{1}{\sigma q_{f \perp}\left(\xi, N_{d}\right)}+i \frac{\Omega_{f}\left(\xi, N_{d}\right) \tau_{m o}}{\sigma q_{f \perp}\left(\xi, N_{d}\right) N_{d}}, \\
& -q_{f z 1}^{\prime \prime}\left(\xi, N_{d}\right)=q_{f z 2}^{\prime \prime}\left(\xi, N_{d}\right) \approx \frac{\Omega_{0} \tau_{m o}}{\sigma q_{0 \perp}\left(\Lambda_{21}+1\right) N_{d}} \frac{\left(\xi / N_{d}+1\right)^{2}}{\xi / N_{d}} .
\end{aligned}
$$

At high intensities, inequality (18a), providing the validity of the results (21a), acquires the form:

$-\frac{\Lambda_{21}}{\Lambda_{21}+1}\left(\frac{s_{2}}{s_{1}}\right)^{2} \gg 1$

As it follows from (21c), results (21a) for the frequency and wave vector are valid, if only the absolute value of $\Lambda_{21}$ is not very small.

Hereinafter the subscript of Glass vectors would be omitted. In fact, when discussing the vector features of the problem it is sufficient to take into account the colinearity of the transverse components of the aforementioned vectors. 


\section{A.N. Morozovska et al.: Dynamics of photoinduced instability in ferroelectric...}

We would like to emphasize that the following scaling exists in (21a). Namely, the values of $\Omega_{f} / N_{d}, q_{f \perp} / N_{d}$ and complex gain $\mathrm{iq}_{\mathrm{fz}}$ depend only over one dimensionless variable $\xi / N_{d}$. The dependences of $\Omega_{f}\left(N_{d} \Omega_{0}\right)$ and $q_{f \perp} /$ $\left(N_{d} q_{0 \perp}\right)$, imaginary and real parts of gain factor $i q_{f z}$ over $\xi / N_{d}$ are depicted in Fig. 1 .

Thus based on (9), we can rewrite general solution of the inner field dynamic components in the form:

$\widetilde{\vec{E}}_{f}(x, y, z, t)=\vec{e}_{z}\left(\left(C_{1} \exp \left(i q_{f z 1} z\right)+C_{2} \exp \left(i q_{f z 2} z\right)\right) \times\right.$

$\left.\times \exp \left(i \Omega_{f} t+i \vec{q}_{f \perp} \vec{r}_{\perp}\right)+c . c.\right)$,

constants $C_{1,2}$ can be determined from (8).
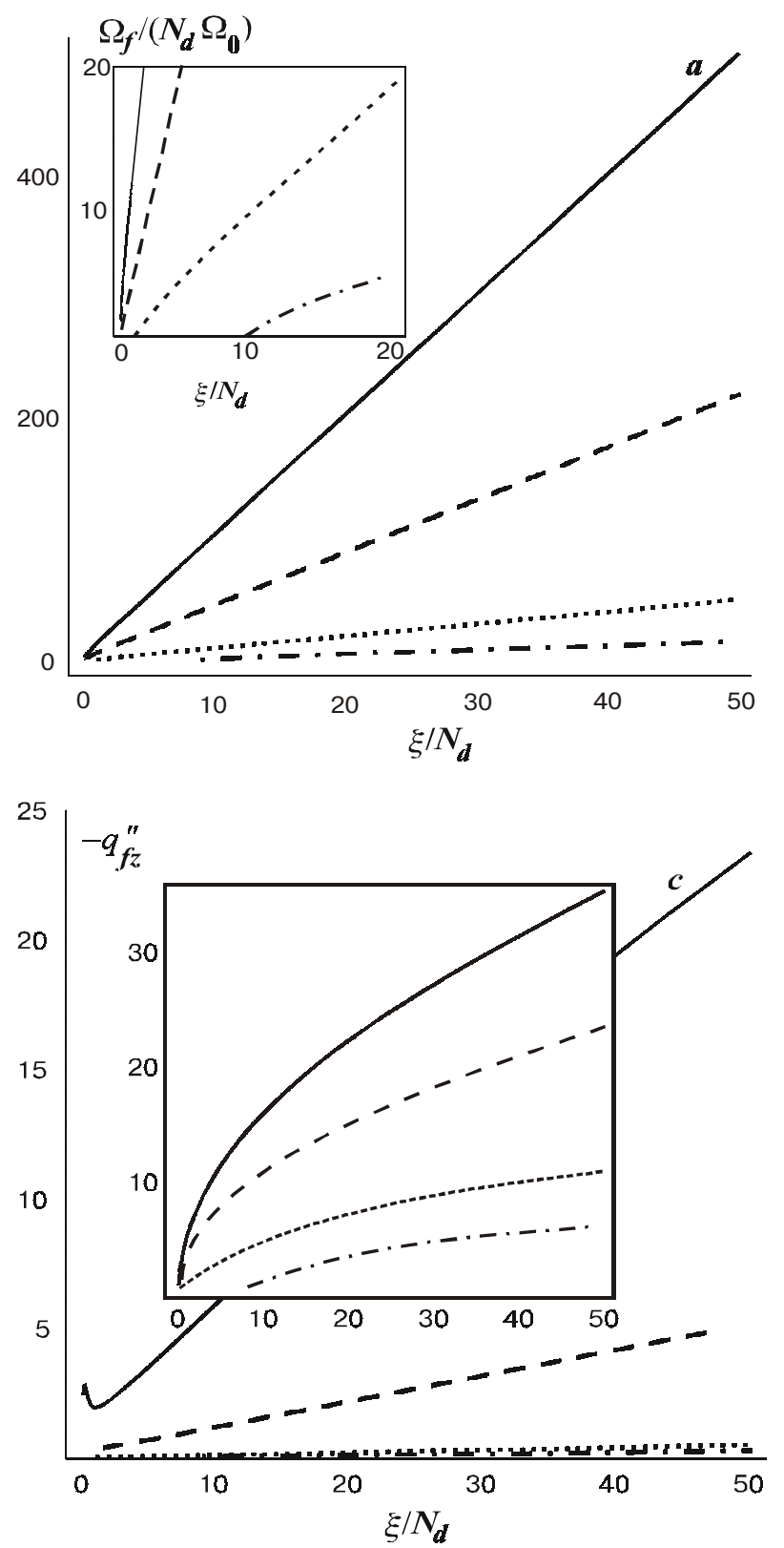

Finally, in accordance with (7-9, 15-16, 186), we can present the solution of the inner field in the form:

$\vec{E}_{f}(x, y, z, t)=\vec{E}_{s}+\vec{e}_{z}\left(U(z) \exp \left(i \Omega \Omega_{f} t+i \vec{q}_{f \perp} \vec{r}_{\perp}\right)+c . c.\right)$,

$U(z)=U_{0}\left(\exp \left(i q_{f z 1} z\right)-\frac{q_{f z 2}}{q_{f z 1}} \frac{\exp \left(i q_{f z 1} \ell\right)-1}{\exp \left(i q_{f z 2} \ell\right)-1} \exp \left(i q_{f z 2} z\right)\right)$

$|U(z)|<<\left|E_{s}\right|, \ell_{c}<z<\ell-\ell_{c}$.

As a consequence, for the concentration of ionized donors one can obtain the following expression:
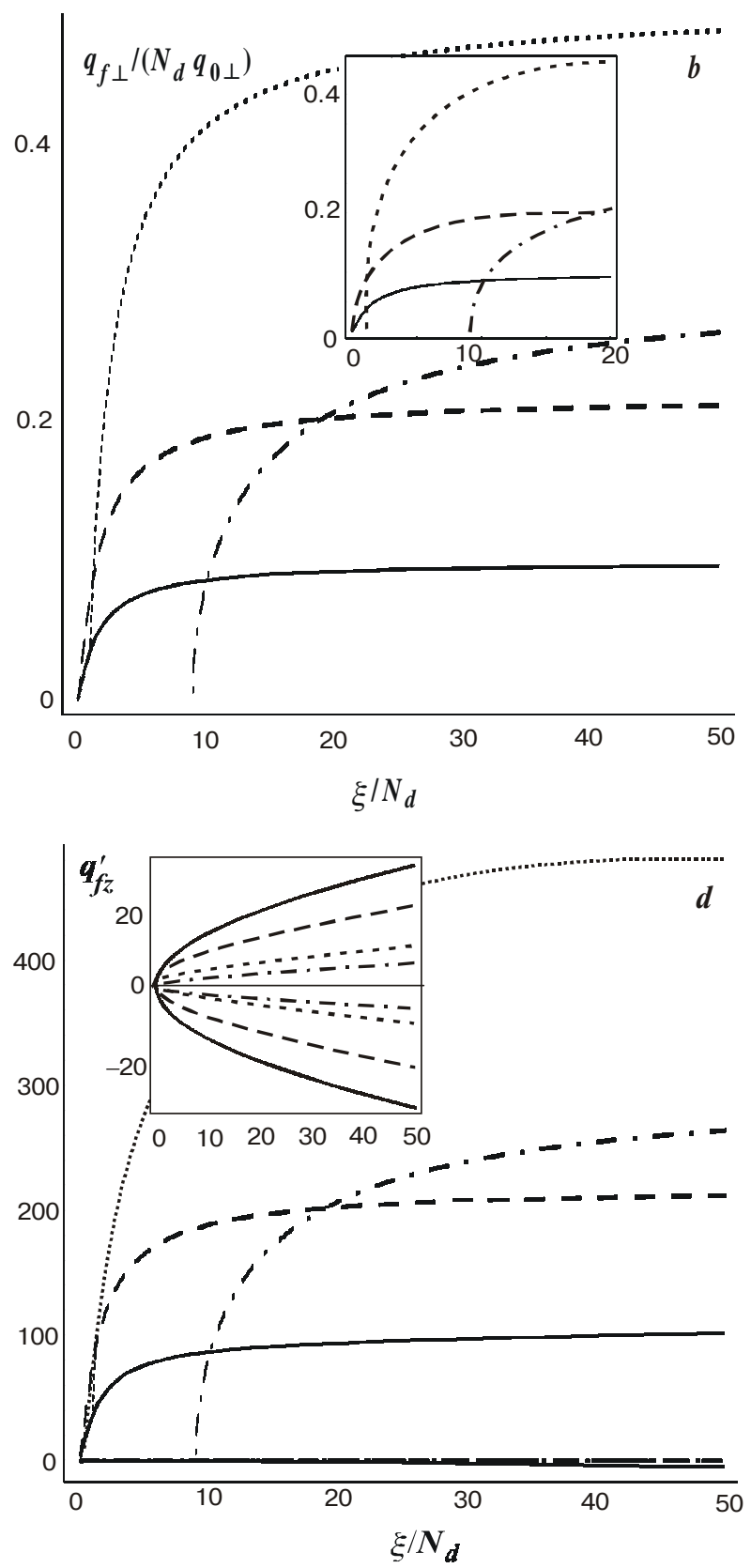

Fig.1. The dependences of $\Omega_{f} f\left(N_{d} \Omega_{0}\right)(a)$ and $q_{f_{\perp}} /\left(N_{d} q_{0 \perp}\right)(b)$, imaginary and real parts of $q_{f z}(c, d)$ on $\xi / N_{d}$ at different $\Lambda_{21}$ values and $\sigma N_{d}=1000, \tau_{m o}=5$ for $(c, d): \Lambda_{21}=-0.1$ (dash-dotted curves); -0.5 (dotted curves); -0.95 (dashed curves); -0.99 (solid curves). In the insets to Figs $(a, b)$ some parts of the aforementioned dependences are shown in details, and in the insets to figs $(c, d)$ the same dependences depicted at $\sigma N_{d}=1$. 


$$
\begin{aligned}
& n_{p}^{+} \approx n_{p s}^{+}\left[1-\frac{\gamma_{p} q_{f z}}{\Omega_{f}^{2}+\left(s_{p} I_{0}+\gamma_{p} n_{e s}\right)^{2}} \frac{i \varepsilon}{4 \pi e} \times\right. \\
& \left.\times\left(U(z) \exp \left(i \Omega_{f} t+i \vec{q}_{f \perp} \vec{r}_{\perp}+i \varphi_{p}\right)+c . c .\right)\right] \\
& \operatorname{tg} \varphi_{p}=\frac{\Omega_{f}}{s_{p} I_{0}+\gamma_{p} n_{e s}} .
\end{aligned}
$$

In accordance with (22c), the recharging trap waves for different donor levels differ not only in amplitude but also in phase.

In (19) the sign "+" must be chosen in expressions (18b) for the frequency and the wave vector. $U_{0} \equiv v\left(q_{f x, y}, q_{f z 1}\right)$ is the Fourier image of fluctuation spectrum (8). Note that in the case of weak diffusion $\left(l_{g}>>l_{d}\right)$ the inequality $\left|q_{f z 2}^{\prime}\right|<<\left|q_{f z 1}^{\prime}\right|$ is valid. Thus, allowing for $q_{f z 2}^{\prime \prime}=-q_{f z 1}^{\prime \prime}$ and $\left|q_{f z 2}^{\prime}\right|<<\left|q_{f z 1}^{\prime}\right|$ one can obtain that $\left|q_{f z 2}\right| \ll<\left|q_{f z 1}\right|$. Therefore, for the "thick" crystal (i.e. at $\left.\left|q_{f z 1,2}^{\prime \prime} \ell\right|>>1\right)$, the expression (19b) for $U(z)$ can be approximated as:

$$
U(z) \approx\left\{\begin{array}{l}
U_{0}\left(\exp \left(i q_{f z 1} z\right)+\frac{q_{f z 2}}{q_{f z 1}} \exp \left(i q_{f z 2}(z-\ell)\right),-q_{f z 2}^{\prime \prime} \ell>>1,\right. \\
U_{0}\left(\exp \left(i q_{f z 1} z\right)-\frac{q_{f z 2}}{q_{f z 1}} \exp \left(i\left(q_{f z 2} z+q_{f z 1} \ell\right)\right),-q_{f z 1}^{\prime \prime} \ell>>1 .\right.
\end{array}\right.
$$

For the "thin" crystal (i.e. at $\left|q_{f z 1,2}^{\prime \prime}\right| \leq 1$ ), the expression (19b) for $U(z)$ can be approximated as:

$$
\begin{aligned}
& U(z)=U_{0}\left(\exp \left(i q_{f z 1} z\right)-\frac{q_{f z 2}}{q_{f z 1}} \exp \left(i q_{f z 2} z\right)\right) \approx \\
& \approx U_{0} \exp \left(i q_{f z 1} z\right) .
\end{aligned}
$$

After standard transformations it becomes clear that the solution (22b) for $U(z)$ in the case of short-circuited sample also coincides with that in the case of the free sample with weak diffusion and bulk fluctuations in the quasi-neutral region of the sample $\ell_{\mathrm{c}}<\mathrm{z}<\ell-\ell_{\mathrm{c}}$.

As the conclusion of this item, we can predict that at sufficient $I_{0}>I_{C}$ and $G_{\perp} \sim \beta_{\perp} \neq 0$ (because $q_{f \perp} \sim l_{g \perp} \sim G_{\perp}$ ), the boundary cycle exists in the nonlinear system (1) in accordance with the Wiener-Hopf theorem. Its spectrum has maximum at $|\Omega|=\Omega_{f}, q_{\perp}= \pm q_{f \perp}$, i.e. the completion degree of traps varies periodically both in time and in space. This means that the stable recharging waves of traps can propagate in the transverse crystal direction.

\subsection{The qualitative analysis of the far field diffracted irradiation. Optimum conditions for the registration of dynamic effects}

This wave creates the modulation of the inner field (22). The latter due to EO-effect records the phase dynamic holographic grating (or lens) with the optical refractive index modulation $\Delta n \sim \sqrt{\left|\tilde{E}_{f}\right|}$. In its turn, the laser pump wave will diffract on this oscillating grating. The following evident speculation permits to determine the features of the diffracted far field structure.

Taking into account (22), the dielectric permittivity modulation has the form:

$$
\begin{aligned}
& \Delta \varepsilon=\varepsilon_{P R}(z)\left(\cos \left(\vec{q}_{f \perp} \vec{r}_{\perp}\right) \cos \left(\Omega_{f} t+\varphi_{f}\right)-\right. \\
& \left.-\sin \left(\vec{q}_{f \perp} \vec{r}_{\perp}\right) \sin \left(\Omega_{f} t+\varphi_{f}\right)\right) .
\end{aligned}
$$

Thus, the aforementioned grating can be treated as the difference of two harmonic two-dimensional phase gratings with the holographic vector $q_{f \perp}$, space shift $\mathrm{p} / 2$ and oscillating amplitudes in accordance with $\cos \left(\Omega_{f} t\right)$ and $\sin \left(\Omega_{f} t\right)$ laws, correspondingly. The diffraction pattern from each grating has two sharp maxima at angles

$\vec{\theta}_{f}=\frac{\vec{q}_{f \perp}}{k_{0}}$.

They look like bright spots, periodically appearing and disappearing with relative intensities $\cos ^{2}\left(\Omega_{f} t\right)$ and $\sin ^{2}\left(\Omega_{f} t\right)$. The superposition of the gratings leads to the oscillations of the maxima in the vicinity of $q_{f}$. The Glass vector rotation and therefore the rotation of the vector $\vec{\theta}_{f} \sim \vec{q}_{f \perp} \sim \vec{G}_{\perp}$ take place due to the pump polarization rotation allowing for photoinduced delay [16]. Thus, the spots transform into periodically running rings or arcs. We would like to stress that the transmitted ("direct") wave consists of the bright central beam and weak (diffracted) side components, whereas the reflected ("inverse") wave has only side components, because our theory is valid if $|U(z)|<<\left|E_{s}\right|$.

Let us discuss the question about the features of laser pump beam necessary for the optimum conditions for the registration of the aforementioned dynamic effects. We would like to remind that the inner field in FRC has been found in the constant pump approximation, i.e. at $I_{0}(x, y, z)=$ const. In fact, we restricted ourselves by the consideration of the rather smooth beam central part. On numerous occasions such beams both theoretically and practically can be modelled by the Gaussian beam:

$$
\begin{aligned}
& A_{0}(x, y, z)=\sqrt{\frac{I_{0}}{1+i n_{f}(z)}} \exp \left(-\frac{\left(x^{2}+y^{2}\right)}{2 \rho_{0}^{2}\left(1+i n_{f}(z)\right)}\right) \\
& n_{f}(z)=\frac{z-z_{0}}{k_{0} \rho_{0}^{2}}
\end{aligned}
$$

where $z_{0}$ is the coordinate of the beam focus. In this case $\left|\Delta \varepsilon\left(r_{\perp}=0\right)\right|>\left|\Delta \varepsilon\left(r_{\perp}=d\right)\right|$, i.e. the $\mathrm{FRC}$ is equivalent to the PR-lens, modulated by bulk holographic grating (23). We will show further that such lens can significantly increase the dynamic PILS intensity in comparison with the case of the pure grating. 


\section{A.N. Morozovska et al.: Dynamics of photoinduced instability in ferroelectric...}

Let us demonstrate this fact for the direct and inverse scattering. Notice that the scattered wave arises deep in the sample under the reflection and scattering of the pump wave on the inner optical inhomogeneities due the refractive index jumps $\delta n=\Delta n_{\mathrm{PRC}}+\Delta n_{\text {dark }}$. Then it reinforces passing through it in the photoinduced-bleaching regime. It can be easily shown that the value of the scattering seeding is proportional to the linear functional of the product $\Delta \varepsilon$ and $\left|A_{0}(x, y, z)\right|[21]$. That is why in order to obtain the highest intensity of a priori weak scattered wave, both the maximum effective value of $\delta e=\Delta \varepsilon_{\mathrm{dark}}+$ $+\Delta \varepsilon_{\mathrm{PRC}}$ and, much more important, the maximum coherence of inverse scattering sources are extremely desirable. In other words, the phase of $\delta \varepsilon$ in the vicinity of different scattering sources must be matched with the pump wave one in such a way to provide the maximum coherence between these sources. This phase matching is impossible for the waves scattered on the dark (growth) imperfections of dielectric permittivity $\Delta \varepsilon_{\text {dark }}$, because $\Delta \varepsilon_{\text {dark }}$ has random structure and no relation with the pump wave. Thus the waves scattered on the dark imperfections almost damp each other and would not be discussed further. For the waves scattered on the photoinduced imperfections of dielectric permittivity $\Delta \varepsilon_{\text {PRC }}$ (see (23)), within the framework of our approximations the phase matching can be substituted by the following condition in the effective scattering $z$-plane: the sum of the direct wave and $\Delta \varepsilon_{\text {PRC }}$ phases does not depend on transverse coordinates. This is possible when the transverse phase profile of the Gaussian pump wave (25) is compensated by the optical phase delay owing to it transmission through the sample with $\Delta \varepsilon_{\text {PRC }}\left(I_{0}\right)$. We'd like to remind that $\Delta \varepsilon_{\mathrm{PRC}}\left(I_{0}\right) \sim I_{0}(x, y, z)$ in accordance with the mechanism of EO-effect (we used that $\ell_{c}<<\ell$ ). It turned out that the condition of the maximum phase matching of inverse scattering waves could be satisfied under the definite restrictions on the initial focus location $z_{0}$. Let us calculate this location by means of the following simplest model.

The real PRC are replaced by the system composed of the thin Gaussian lens with the main plain at $z=0$, which can significantly change only the phase of the incident beam, and of the plane mirror at $z \approx l$ with reflection coefficient $R$. The transfer function of the lens $T(x, y)[26]$ can be exactly obtained from the geometrical speculations, allowing for the expression (see (22b)) for $\Delta n_{\mathrm{PRC}}\left(I_{0}\right)$ :

$\Delta n_{P R C}\left(I_{0}\right)=\chi I_{0}(x, y, z)\left(1+v(z) \exp \left(i \Omega_{f} t+i \vec{q}_{f \perp} \vec{r}_{\perp}\right)+\right.$ c.c. $)$,

$v(z)=\frac{U(z)}{U_{0}}$.

Also $\left|\Delta n_{\mathrm{PRC}}\left(I_{0}\right)\right|<<1$ in agreement with our assumptions about the validity of the perturbation theory. Having calculated the optical phase delay one can derive that:

$$
\begin{aligned}
& T(x, y) \approx \exp \left[i k_{0} \chi I_{0} \int_{0}^{\ell} \exp \left(-\frac{\left(x^{2}+y^{2}\right)}{\rho_{0}^{2}\left(1+n_{f}^{2}(\xi)\right)}\right) \frac{d \xi}{1+n_{f}^{2}(\xi)}\right] \\
& \left(1+i k_{0} \chi I_{0} \int_{0}^{\ell} \frac{v(z) \exp \left(i \Omega f t+i \vec{q}_{f \perp} \vec{r}_{\perp}\right)+c . c .}{1+n_{f}^{2}(\xi)} d \xi\right) \approx \\
& \approx \exp \left[i k_{0} \chi I_{0} \ell\right] \exp \left[-i k_{0} \chi I_{0} \frac{\left(x^{2}+y^{2}\right)}{\rho_{0}^{2}} \int_{0}^{\ell} \frac{1}{\left(1+n_{f}^{2}(\xi)\right)^{2}} d \xi\right] .
\end{aligned}
$$

The amplitude of the laser far field at $z= \pm L$, i.e. after single (direct PILS) and double (inverse PILS) passing through the PR-lens can be obtained after the Kirchhoff transformation as follows:

$$
\begin{aligned}
& E_{1}\left(x_{p}, y_{p}, z_{p}>\ell\right)= \\
& =\frac{k_{0}}{z_{p}} \int_{-\infty}^{\infty} d x d y T(x, y) A_{0}(x, y, 0) \exp \left(i k_{0}\left|\vec{r}_{p}-\vec{r}\right|\right), \\
& E_{2 R}\left(x_{p}, y_{p}, z_{p}<<0\right)= \\
& =R \frac{k_{0}}{z_{p}} \int_{-\infty}^{\infty} d x d y T^{2}(x, y) A_{0}(x, y, 0) \exp \left(-i k_{0}\left|\vec{r}_{p}-\vec{r}\right|\right),(26) \\
& \text { if } \frac{k_{0} \rho_{0}^{2}}{z_{p}}<<1,\left|\vec{r}_{p}-\vec{r}\right| \approx z_{p}+\frac{\left(x-x_{p}\right)^{2}+\left(y-y_{p}\right)^{2}}{2 z_{p}} .
\end{aligned}
$$

Analyzing the integrants in (26c), the conditions of the maximum phase matching can be reduced to the absence of transverse-dependent phase $\Psi$ of the functions $T(x, y) A_{0}(x, y, 0)$ and $T^{2}(x, y) A_{0}(x, y, 0)$ :

$$
\begin{aligned}
& \Psi_{1}\left[T(x, y) A_{0}(x, y, 0)\right] \approx 0 \Leftrightarrow \\
& n_{f}(0)=\int_{0}^{\ell} \frac{2 k_{0} \chi I_{0}}{\left(1+n_{f}^{2}(z)\right)^{2}} d z \approx \frac{2 k_{0} \ell n_{P R C}^{S}\left(I_{0}\right)}{\left(1+n_{f}^{2}(0)\right)^{2}}, \\
& \Psi_{2 R}\left[T^{2}(x, y) A_{0}(x, y, 0)\right] \approx 0 \Leftrightarrow \\
& n_{f}(0)=\int_{0}^{\ell} \frac{4 k_{0} \chi I_{0}}{\left(1+n_{f}^{2}(z)\right)^{2}} d z \approx \frac{4 k_{0} \ln n_{P R C}^{S}\left(I_{0}\right)}{\left(1+n_{f}^{2}(0)\right)^{2}}
\end{aligned}
$$

Expressions (26d) can be simplified for small $n_{f}(0)$. In this case both for direct and inverse PILS one can obtain:

if $\left|k_{0} \ell \chi I_{0}\right|<<1, \quad z_{0} \sim \Delta n_{P R C}^{S}\left(I_{0}\right) k_{0}^{2} \rho_{0}^{2} \ell$

As it follows from (26d), if $\Delta n_{P R C}^{S}<0$, the pump beam focus must be located in front of the PRC, and if $\Delta n_{P R C}^{S}>0$, the pump beam focus must be located inside the PRC.

SQO, 5(3), 2002 


\section{A.N. Morozovska et al.: Dynamics of photoinduced instability in ferroelectric...}

Hence, in order to register the aforementioned PILSeffects successfully, it is desirable to use definitely focused (but not the plane) laser pump beams.

All material parameters of the crystal have been regarded as constants. Effectively, they vary because of the aforementioned polarization vector rotation and such growth peculiarities as non-homogeneous impurity distribution (i.e. the fluctuations of $n_{d}$ ), the floating of the crystallographic axes $\mathrm{X}$ due to the local symmetry breaking near defects (i.e. the fluctuations of $G_{\perp}$ ) [16]. That is why the whole set of frequencies and wave vectors are present in the real system. The final results must be averaged over all fluctuating values within the framework of the definite non-homogeneities model. For our theory this result is the laser field changed by interaction with the $\mathrm{PRC}$ in the far zone from the crystal, i.e. the diffraction pattern on a screen.

As the final remark to this item, we would like to emphasize the following. Our theory predicts that high quality ferroelectric photorefractive ilmenites, in the case when a photo-active impurity does not destroy their initial structure, can generate optical autowaves even under stationary laser irradiation.

\subsection{The discussion and analysis of the temporal and spatial characteristics of the scattered irradiation. Comparison with the experiment on autowave PILS in $\mathrm{LiNbO}_{3}: \mathrm{Fe}$}

In this item, we restrict ourselves by calculation and analysis of those basic characteristics of inverse scattering, which have been compared with the available experimental data on autowave scattering in $\mathrm{LiNbO}_{3}$ crystals doped with $0.02 \div 0.07 \mathrm{wgh} . \%$ Fe revealed before [6] and intensively studied now in our lab.

Hereinafter in this item, we assume that it is possible to neglect the dark conductivity due to sufficient laser intensity $s_{p} I_{0} \gg s_{0}$, i.e. to substitute $s_{0}=0$.

We would like to remind that aforementioned inverse scattering has the conic spatial structure with the cone opening oscillating around $\theta_{f}$ (see commentary to (2224)). It is appeared convenient to characterize these temporal oscillations by the minimum angle $\theta_{\text {min }}$ (i.e. the angle of cone generation), the angle of the best visualization $\theta_{0}$ (i.e. the angle of cone maximum intensity), and the maximum angle $\theta_{\max }$ (i.e. the angle of cone disappearance). The experimentally measured values of $\theta_{\max }$ and $\theta_{\min }$ can be additionally limited by the resolution of the register hardware. We determined these angles by numerical simulation of Gaussian beam diffraction [17] at the diffraction grating (23). It turns out that for a wide region of parameters with high accuracy one can apply clear and simple relations:

$$
\begin{aligned}
& \theta_{0} \approx\left(\theta_{f \max }+\theta_{f \min }\right) / 2 \approx \theta_{f}, \\
& \theta_{f \text { min }} \approx H_{1} \theta_{f}, \\
& \theta_{f \text { max }} \approx H_{2} \theta_{f} .
\end{aligned}
$$

Where $H_{1}<1, H_{2}>1$ are positive constants. Thus, the cone parameters are determined by the angle $\theta_{f}$, which increases with increasing the photo-active impurity concentration $n_{d}$ (in $\%$ ), because $n_{d}=$ const $\cdot N_{d}$. Finally from $(9,21)$ we obtain:

$\theta_{f}\left(\xi, n_{d}\right)=$

$=\theta_{g} n_{d} \frac{\xi / n_{d}}{\xi / n_{d}+1} \sqrt{-\left(\Lambda_{21}+1\right)\left(\Lambda_{21}+1 /\left(\xi / n_{d}+1\right)\right)}$.

Here $\theta_{g}$ does not depend on $n_{d}$, and dimensionless intensity $\xi \sim I_{0}$ and $-1>\Lambda_{21}>0$ are the fitting parameters. Calculated in accordance with (27-28) dependences of the angles $\theta_{\min }, \theta_{0}$ and $\theta_{\max }$ over impurity concentration $\mathrm{n}_{\mathrm{d}}$ have been compared with the experimentally obtained ones in Fig.2.

Let us discuss the temporal spectrum of scattering waves. The theoretical (see Eq.(21)) and experimental dependences of the autowave frequency $\Omega_{f}$ over the pump intensity $I_{0}$ are presented in Fig.3. To all appearances, we possess the experimental data for the linear part of the aforementioned dependence, i.e. for high-quality samples $\left(n_{d} \approx\right.$ const) and rather high $I_{0}$ (see Fig. $1 a$ and Eq.(21)). The investigation of the process at smaller intensities proves the existence of an intensity threshold: the autowave PILS loses its periodical structure and eventually splits into random flickering spots at intensities smaller that the definite for each sample critical intensity $I_{C}$. Within the framework of our theory for $I_{0}>I_{C}$ the autowave frequency does not depend on the crystal thickness. Possibly, this dependence is present below the intensity threshold. The temporal dependence of the autowaves intensity averaged over ring can be easily calculated when using the theory of mathematical diffrac-

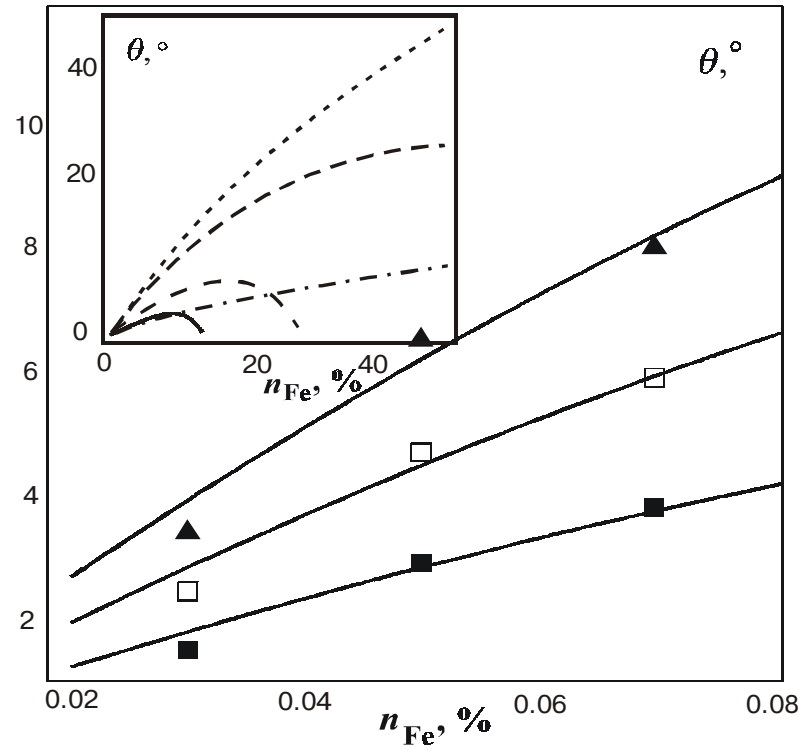

Fig.2. The dependences of $\theta_{\min }$ (filled squares), $\theta_{0}$ (empty squares), $\theta_{\max }$ (empty triangles) on Fe concentration in $\mathrm{LiNbO}_{3}$. The best agreement with the experiment was obtained at $\xi=2.5$, $\Lambda_{21}=-0.1, \theta_{g}=340^{\circ}, H_{1}=0.632, H_{2}=1.382$. The dependence of the ratio $\theta_{0}$ on $n_{d}$ at different $\Lambda_{21}$ is shown in the inset: $\Lambda_{21}=-0.99$ (dash-dotted curve), (dotted curve), -0.3 (short-dashed curve), -0.1 (long-dashed curve), -0.01 (solid curve). 


\section{A.N. Morozovska et al.: Dynamics of photoinduced instability in ferroelectric...}

tion [18]. In comparing the result with the experimentally obtained autowaves evolution (see inset in Fig.3), to our mind, the qualitatively compliance in general dynamics of the process is evident, the differences of the experimental and theoretical profiles on the period is related with the linearity of the theory. In fact, we omitted all nonlinear terms in the initial system, i.e. neglected the possibility of the crystal to generate sub- and super-harmonics.

Let us calculate such important characteristic as the spatial correlation coefficient of the inverse scattering, hereinafter designated as $K_{a b}$. Namely, let us derive the correlation function of the two points " $a$ " and " $b$ " on the screen (characterized by angles $\theta_{a}$ and $\theta_{b}$ ) with different (in general) time-averaged intensities $j_{2}\left(\vec{\theta}_{a}\right)=\left\langle\left\langle j_{2}\left(\vec{\theta}_{a}, \tau\right)\right\rangle\right\rangle_{\tau}$ and $j_{2}\left(\vec{\theta}_{b}\right)=\left\langle\left\langle j_{2}\left(\vec{\theta}_{b}, \tau\right)\right\rangle\right\rangle_{\tau}$. By definition,

$K_{a b}=\frac{2 j_{2}\left(\vec{\theta}_{a}\right) j_{2}\left(\vec{\theta}_{b}\right)}{\left(j_{2}\left(\vec{\theta}_{a}\right)\right)^{2}+\left(j_{2}\left(\vec{\theta}_{b}\right)\right)^{2}}$.

In fact, we calculate $\mathrm{K}_{\mathrm{ab}}$ for two different "flickering spots" or parts of "flickering arcs" or points of "irregular rings". Within the framework of the models of nonhomogeneities proposed in our previous works [21], the conic cross-sections with various vectors $\theta_{f a}, \theta_{f b}$ and amplitudes $R_{a}, R_{b}$ can be realized in the points " $a$ " and " $b$ ". These amplitudes $R_{a, b}$ do not depend on the angles $\theta_{f a}$ and $\theta_{f b}$. For the case, when side maxima are good resolved (i.e. $\left(\eta \theta_{f}\right)^{2}>>1$ ) and

$\theta_{a} \approx \theta_{b} \approx \theta_{f a} \approx \theta_{f b}$,

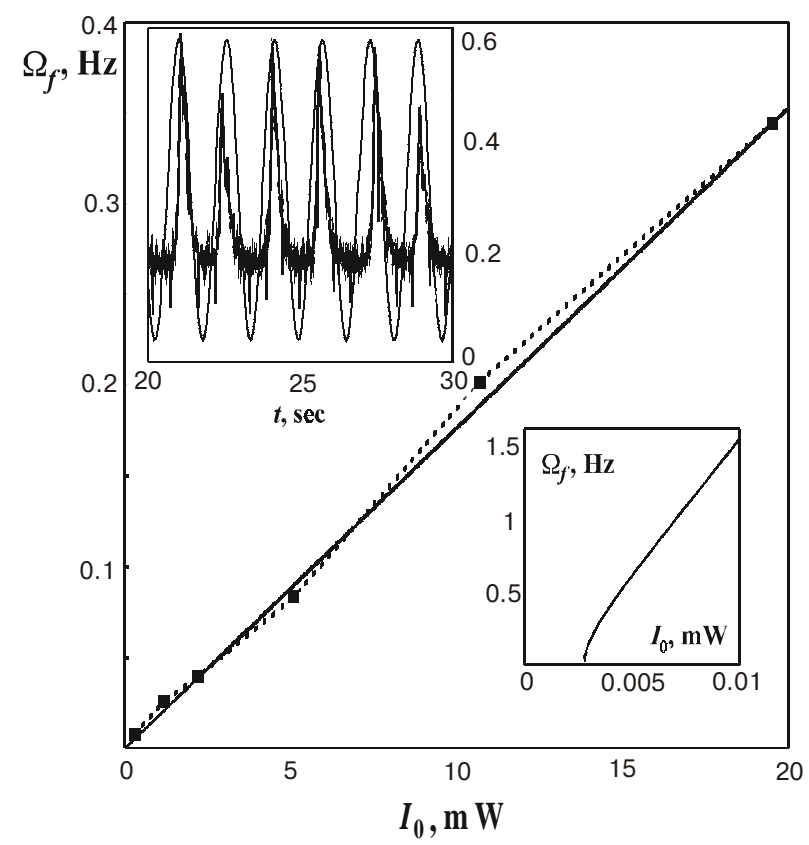

Fig.3. Theoretical (solid curve, calculated by Eq.(21a) at $\Lambda_{21}=-0.1$, $s_{1}=0.053 \mathrm{~mJ}^{-1}, N_{d}=0.03 \%$ ) and experimental (filled squares) dependencies of autowaves frequency $\Omega_{f}$ on $I_{0}$. Upper inset: the experimental (bold curve) and theoretical (fine curve) dependences of the averaged over the ring autowave intensity (in arbitrary units) on time. Lower inset: theoretical dependence of autowaves frequency $\Omega_{f}$ on $I_{0}$ in the vicinity of the threshold at the same fitting parameters.
Equation (29) can be essentially simplified and rewritten as the function of dimensionless intensity $\xi$, introduced in item 2.3. In accordance with the numerical calculations (see Fig.1b), even at pump intensities a little larger than $\mathrm{I}_{\mathrm{C}}$, the expression (28) can be used for $\theta_{f}$. Using (28) and (30), one can obtain that

$\theta_{a} \approx \theta_{b} \approx \theta_{f a} \approx \theta_{f b}=$ $=\theta_{0} N_{d} \frac{\xi / N_{d}}{\xi / N_{d}+1} \sqrt{-\left(\Lambda_{21}+1\right)\left(\Lambda_{21}+1 /\left(\xi / N_{d}+1\right)\right)}$.

Therefore, $K_{a b}$ can be presented as:

$$
K_{a b}(\xi)=\frac{2 K_{0} \exp \left(\frac{\xi_{0}\left(\Lambda_{21}+1 /\left(\xi / N_{d}+1\right)\right)}{\left(N_{d} / \xi+1\right)^{2}}\right)}{1+K_{0}^{2} \exp \left(2 \frac{\xi_{0}\left(\Lambda_{21}+1 /\left(\xi / N_{d}+1\right)\right)}{\left(N_{d} / \xi+1\right)^{2}}\right)},
$$

$\xi=s I_{0} / \gamma n_{0}$,

$\xi_{0} \approx N_{d}^{2} \eta^{2}\left(\Lambda_{21}+1\right)\left(\operatorname{Cos}\left(\angle \vec{\theta}_{a}, \vec{\theta}_{f a}\right)-\operatorname{Cos}\left(\angle \vec{\theta}_{b}, \vec{\theta}_{f b}\right)\right)$

$-1<\Lambda_{21}<0$.

$\xi>\xi_{C}=-N_{d}\left(1+\frac{1}{\Lambda_{21}}\right)$

The dependence of the spatial correlation coefficient $K_{a b}$ over $\xi / N_{d}$ and comparison with recently obtained

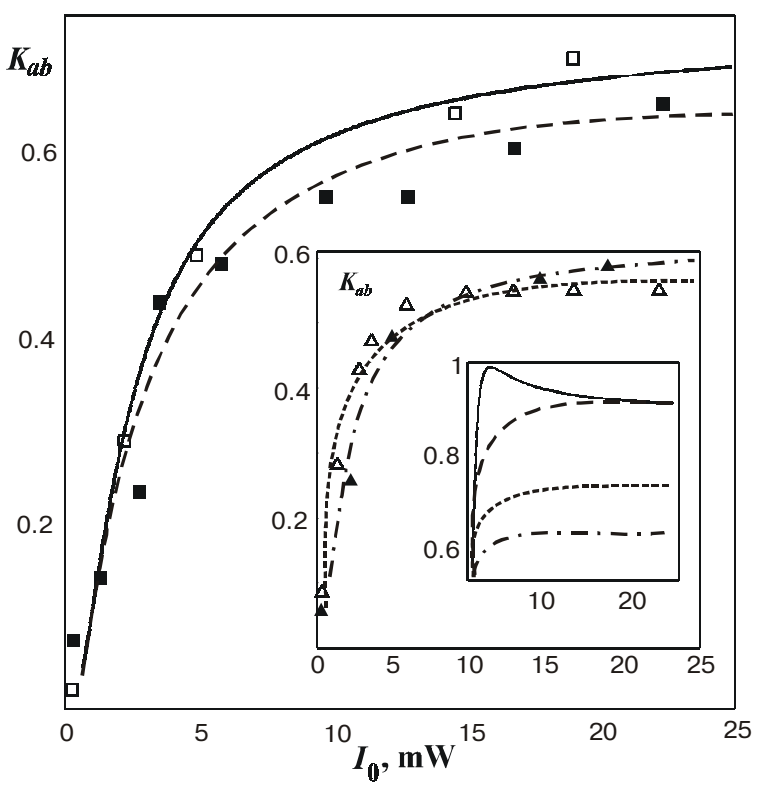

Fig.4. The dependences of the measured and calculated at $\Lambda_{21}=-0.1$ and $I_{0 \mathrm{~K}}=0.07 \mathrm{~mW}$ spatial correlation coefficient $K_{a b}$ on the intensity $I_{0}$ at the different angles between the detectors: $10^{\circ}$ (empty squares and solid curve at $K_{0}=0.021, \xi_{0}=-30.59 \mathrm{~mW}$ ), $30^{\circ}$ (filled squares and dashed curve at $K_{0}=0.019, \xi_{0}=-30.59$ $\mathrm{mW}), 90^{\circ}$ (empty triangles and dotted curve at $K_{0}=0.09, \xi_{0}=-$ $12.74 \mathrm{~mW}$ ), $180^{\circ}$ (filled triangles and dash-dotted curve at $K_{0}=$ $\left.0.0356, \xi_{0}=-22.94 \mathrm{~mW}\right)$. The dependence of $K_{a b}$ over $I_{0}$ at $K_{0}=$ 0.3 and different $\xi_{0}$ is depicted in the inset: $\xi_{0}=-2.08 \mathrm{~mW}$ (dashdotted curve); $-4.16 \mathrm{~mW}$ (dotted curve); $-8.32 \mathrm{~mW}$ (dashed curve); $-16.64 \mathrm{~mW}$ (solid curve).

SQO, 5(3), 2002 


\section{A.N. Morozovska et al.: Dynamics of photoinduced instability in ferroelectric...}

experimental data are represented in Fig.4. Then, in order to obtain the dependence of $K_{a b}$ over pump intensity $\mathrm{I}_{0}$, it is sufficient to make the substitution $\xi / N_{d} \rightarrow I_{0} / I_{0 \mathrm{~K}}$ in (31). In the case, the values $\xi_{0}, I_{0 K}$ and $K_{0}$ are fitting parameters. If the parameter $\Lambda_{21}$ could not be found from the critical intensity value or other experimental data, it also could be regarded as the fitting one. Without further limitations one can assume that $0<K_{0}<1$ and $\xi_{0}$ can be both positive and negative. Under comparing the dependence $K_{a b}$ on $I_{0}$ with the experiment, we used (31) and, therefore, considered the register detectors placed on the ring in accordance with (30) assuming that they were the point ones (i.e., we approximate the real apparatus function by the Dirac delta-function). The latter approximation is pertinent only if $\theta_{d}<<\left|\vec{\theta}_{b}-\vec{\theta}_{a}\right|\left(\theta_{d}\right.$ is the angular detector size).

We would like to stress that autowave PILS was observed only in the case of the focused (but not the plane) Gaussian pump beam with the beam focus located directly in front of the PRC. This fact is in a good agreement with the conclusions of the item 2.4 (see (26d)).

In our laboratory all the above-mentioned dynamic effects (see Fig.2) have been registered only in the highquality $\mathrm{LiNbO}_{3}$ single crystals doped with Fe. Similar effects have not been found in any other samples. In accordance with the conclusion of the item 2.3, we explain these results by the presence of the non-zero component $\mathrm{G}_{\wedge}$ only in the ilmenite $\mathrm{LiNbO}_{3}$ and its absence in all other investigated samples.

The process of autowave PILS by crystal strongly depends on a laser light wavelength. Namely, autowaves are almost absent at wavelengths more than $0.44 \mu \mathrm{m}$. This fact can be explained by the extremely sharp (actually threshold) dependence of the such material parameters as photoionization and capture coefficients as well as PV-tensor coefficients on the energy of quanta absorbed by PR-medium, i.e. over laser irradiation wavelength. Really, the light quantum must have the sufficient energy for the donor ionization and for the photoelectron casting to the conduction band.

Having analyzed all the aforementioned results we can formulate the following statement concerning the origin and structure of donor levels in $\mathrm{LiNbO}_{3}: \mathrm{Fe}$. Both of the donor levels are made of iron atoms $\mathrm{Fe}^{+2}(\mathrm{I})$ and $\mathrm{Fe}^{+2}(\mathrm{II})$, which substitute lithium $\mathrm{Li}^{+1}$ and $\mathrm{Nb}^{+5}$ atoms as $\mathrm{Fe}^{+3}(\mathrm{I}, \mathrm{II})$ centers, correspondingly. Really, these $\mathrm{Fe}^{+3}$ centers occupy different cation sites in pairs to keep the charge equilibrium. This conclusion is confirmed by other independent investigations [23-25]. The $\mathrm{Fe}^{+3}$ trap-center can be transformed into $\mathrm{Fe}^{+2}$ donor-center after special preparation of the sample, such as heating, annealing, or preliminary laser irradiating. In fact, autowave PILS exists only in as-prepared samples.

Thus, though the real temporal spectrum of PILS has been approximated by the delta-function in our linearized theory, the main spatial and temporal characteristics of the process obtained in this way are in a full agreement with the available experimental data.

\section{Generalization of results. The master equation for inner field evolution}

It is worth to emphasize that the main result of the paper is the running holographic grating (20), obtained from the microscopic equations (1) in the item 2. All the peculiarities of PILS by the grating can be easily described qualitatively (see the end of item 2.3) and obtained by standard methods of diffraction theory [18]. In order to generalize the obtained results over more wide class of systems, we would like to formulate the following problem.

It is desirable to derive and to analyze the phenomenological equation for inner field dynamics by synergetic methods, the Fourier- and Laplace-images of which have the same structure as (12), but the material constants have the most general form. Then, instead of the specific initial system (1), the general equation for inner field can be postulated. The behaviour of its coefficients at the instability threshold almost determines the system dynamics. Using H. Haken terminology [19] hereinafter we regard the equation as master equation.

\subsection{Master equation}

Let us discuss a semiconductor PR-medium with local (e.g., usual conductivity, bias, photovoltaic) and nonlocal (e.g., diffusion) mechanisms of carrier transfer. The main features of its zone structure with respect to carriers releasing under light illumination can be described by some many-level system (e.g., a conductive band and ionized donor levels) with the concentration of charge carriers in the conductive band $N_{c}$ and the concentration of $p$ type donor atoms $N_{p}$. The density of each type of material current is proportional to the concentration of definite carriers.

After standard but cumbersome transformations, the initial system (1) can be reduced to that of equations describing the inner electric field $E_{f}$ and the dynamics of carrier concentration $N_{c}$. Suppose that the system can be linearized near the stationary points. The existence of such points hereinafter is postulated.

Really, one can linearize it in the vicinity of the some stationary point $\vec{E}_{f}=\vec{E}_{s}+\varepsilon_{f}(x, y, z, t), N_{c}=$ $=N_{c s}+\tilde{n}(x, y, z, t)$, and after standard transformations obtain the linearized equation for $\varepsilon_{f}$. Let us generalize and simplify this equation in such a way that its structure appears to be the clearest and the number of parameters becomes minimum and varying in the widest range. The following equation satisfies all the aforementioned conditions:

$$
\begin{aligned}
& \left(1+\frac{\partial}{\partial \tau}\right) \varepsilon_{f}+\operatorname{grad}_{\zeta}\left(\operatorname{div}_{\zeta}\left(\varepsilon_{f}\right)\right)+ \\
& +\sum_{p} \vec{\Lambda}_{p}\left(\operatorname{div}_{\zeta}\left(\varepsilon_{f}\right)+\int \tilde{\int} \tilde{t} \operatorname{div}_{\zeta}\left(\varepsilon_{f}(\tilde{t})\right) m_{p} \exp \left(-F_{p}(\tau-\tilde{t})\right)\right)=0 .
\end{aligned}
$$




\section{A.N. Morozovska et al.: Dynamics of photoinduced instability in ferroelectric...}

Here the dimensionless variables are introduced: time $\tau=t / \tau_{m}$, radius vector $\vec{\zeta}=\vec{r} / \ell_{d}$ and constants: the relative drift length $\vec{\Lambda}_{p} \sim \vec{\ell}_{g p} / \ell_{d}, m_{p}=\tau_{m} / \tau_{p}, F_{p}=f_{p} \tau_{m} / \tau_{p}$ $\left(f_{p}>1\right)$. Other designations used here are the same as in (12), in this item we consider them as real constants (all positive except $l_{\mathrm{g}}$ ).

It will be proved that the obtained equation (32) for the renormalized inner field is the master equation. It coincides with the one investigated in details in references [7-9,20] at $m_{p}=0$ or $F_{p}=\infty$. Let us discuss the sense of each term in (32). Essentially, this equation represents the conservation law of the net current in the system. Indeed, the term $(1+\partial / \partial \tau) \varepsilon_{f}$ is the bias and conduction current, the term $\operatorname{grad}_{\vec{\zeta}}\left(\operatorname{div}_{\vec{\zeta}} \varepsilon_{f}\right)$ is a generalized non-local (diffusion) current, and the last term $(1-C \partial / \partial \tau) \vec{\Lambda} \operatorname{div}_{\vec{\zeta}} \vec{\varepsilon}$ is the local (photovoltaic) photocurrent. Notice that $F_{p}$ are not zero in the general case of model two-level system of sluggish PR-medium with a non-local response and asymmetrical potential of photoactive impurity centers.

\subsection{The analysis of the spatial-temporal system spectrum}

The (32) can be solved by the method of integral transformations (as (9)), i.e.

$\varepsilon_{f}(\vec{\zeta}, \tau)=\int d \vec{q} d \Omega \cdot \vec{U}(\vec{q}, \Omega) \exp (i \vec{q} \vec{\zeta}+i \Omega \tau)$

where $\vec{q} \vec{\zeta}=q_{\mid} \zeta_{z}+\vec{q}_{\perp} \vec{\zeta}_{\perp}$. Thus, the equation for the image has the form:

$\vec{U}(1+i \Omega)-i(\vec{q} \vec{U}) \times\left(i \vec{q}-\sum_{p=1,2} \vec{\Lambda}_{p}\left(1-\frac{m_{p}}{i \Omega+F_{p}}\right)\right)=0$

Again, let us generalize and simplify this equation in such a way that its structure appears to be the clearest and the number of parameters becomes minimum and varying in the widest range.

In the case, when $F_{1}>>F_{2}, m_{1} \sim m_{2}$ and vectors $\vec{\Lambda}_{p}$ are co-linear, (34) can be reduced to the more simple differential equation with real scalar parameters $B, C$ and a real vector parameter $\Lambda$, which satisfies all the aforementioned conditions and can be rewritten as:

$(1+i \Omega) \vec{U}+\left(\vec{q}+\frac{i-\Omega C}{1+i \Omega B} \vec{\Lambda}\right)(\vec{q} \vec{U})=0$.

Here:

$$
\begin{aligned}
& B=\frac{1}{F_{1}}, \quad C=\frac{1+\Lambda_{2} / \Lambda_{1}}{F_{1}\left(1-m_{1} / F_{1}+\Lambda_{2} / \Lambda_{1}\right)}, \\
& \vec{\Lambda}=\vec{\Lambda}_{1}\left(1-\frac{m_{1}}{F_{1}}\right)+\vec{\Lambda}_{2} .
\end{aligned}
$$

In order to lock the problem, it is necessary to establish the dependences of coefficients in (35b) on the pump beam intensity $I_{0}$. In accordance with general speculations, we propose the following dependences:

$$
\begin{aligned}
& B\left(I_{0}\right)=\frac{1}{\tau_{m} s I_{0} F\left(I_{0}\right)}, \\
& C=B\left(I_{0}\right) \frac{\Phi}{\Phi-1 / F\left(I_{0}\right)}, \\
& F\left(I_{0}\right)=1+\frac{I_{f}}{I_{0}}, \tau \sim t / \tau_{m}, \\
& \vec{\Lambda}=\vec{\Lambda}_{0}\left(\Phi-1 / F\left(I_{0}\right)\right), I_{f}>0 .
\end{aligned}
$$

After supplementing (35) by the wave equation for pump field taking into consideration the EO-effect, we obtain the complete system of the nonlinear equations for the description of PILS in PRC. As before, we restrict ourselves by solving (35) in the case of constant coefficients, i.e. at $I_{0}=$ const.

Under the conditions of the absence of the tangential (transverse) components of the inner field

$\varepsilon_{f \perp}(\vec{\zeta}, \tau)=0$.

when substituting (35d) in (32), the following relations can be obtained:

$\vec{q}_{\perp}=\frac{-i+\Omega C}{1+i \Omega B} \vec{\Lambda}_{\perp}$,

$q_{\|}^{2}+\frac{i-\Omega C}{1+i \Omega B} \Lambda_{\|} q_{\|}+1+i \Omega=0$.

Hereinafter we consider the system with a non-zero transverse photovoltaic current, i.e. $\Lambda_{\perp} \neq 0$. The absolute instability threshold of the system, infinite in transverse and limited in longitudinal directions, is determined by the equalities:

$\operatorname{Im}(\Omega)=\operatorname{Im}\left(\mathrm{q}_{\perp}\right)=0$.

Substituting (37) into (36) one obtains:

$$
\begin{gathered}
\Omega= \pm \frac{1}{\sqrt{-B C}}, \quad \vec{q}_{\perp}= \pm \sqrt{-\frac{C}{B}} \vec{\Lambda}_{\perp}, \\
q_{\|}^{2} \mp \sqrt{-\frac{C}{B}} \Lambda_{\|} q_{\|}+1 \pm \frac{i}{\sqrt{-B C}}=0 .
\end{gathered}
$$

As it follows from $(36,38 \mathrm{~b})$, two values of longitudinal wave vector, which determines the spatial gain factor, correspond to the each frequency value (38a), i.e.:

$$
\begin{aligned}
& q_{1}= \pm \sqrt{-\frac{C}{B}} \frac{\Lambda_{\|}}{2}-\sqrt{\frac{|C|}{4 B} \Lambda_{\|}^{2}-1 \mp \frac{i}{\sqrt{-B C}}} \\
& q_{2}= \pm \sqrt{-\frac{C}{B} \frac{\Lambda_{\|}}{2}+\sqrt{\frac{|C|}{4 B} \Lambda_{\|}^{2}-1 \mp \frac{i}{\sqrt{-B C}}}} .
\end{aligned}
$$




\section{A.N. Morozovska et al.: Dynamics of photoinduced instability in ferroelectric...}

The signs in (38) must be chosen simultaneously upper or lower.

In accordance with (38), such transverse instability as the Hopf bifurcation can be realized in the systems with a non-local response $\left(l_{d} \neq 0\right)$ where:

$B C<0$ и $\Lambda_{\perp} \neq 0$.

In the terms of model system (35b) the condition (39a) means that $C<0$. The latter is possible $-1<\Lambda_{2} / \Lambda_{1}<-1+m_{1} / F_{1}$. When using (35c), one can determine that $C<0$ if

$I_{0}>I_{C}, \quad I_{C}=\frac{I_{f}}{1 / \Phi-1}, \quad 0<\Phi<1$.

Actually, this means that the process possesses the threshold over pump intensity. All aforementioned nonlinear phenomena are absent or uncorrelated due to the vanishing gain below the intensity threshold (39b), they become noticeable only above this threshold. This means that the dynamic properties of specific system (1) possess the aforementioned threshold, i.e. they can be registered in the samples with rather developed photovoltaic response at the pump intensities above the critical value.

When using $(31,35)$, the renormalized inner field can be rewritten as:

$\varepsilon_{f}(\vec{\zeta}, \tau)=\vec{e}_{z}\left(\left(C_{1} \exp \left(i q_{1} \zeta_{z}\right)+C_{2} \exp \left(i q_{2} \zeta_{z}\right)\right) \times\right.$

$\exp \left(i \Omega \tau+i \vec{q}_{\perp} \vec{\zeta}_{\perp}\right)+$ c.c. $)$

where coefficients $C_{1,2}$ can be determined from the appropriate boundary conditions for the longitudinal inner field.

Let us discuss the dependences of oscillations frequency $\Omega_{f}=\Omega / \tau_{m}$, of the transverse wave vector $Q=q_{\perp} / l_{d}$ and of the spatial gain factor $g=q_{\|} / l_{d}$ on the pump intensity $I_{0}$ for the dynamic holographic grating recorded in the medium by inner field (40). Allowing for (35c) we can obtain from (38) that:

$$
\begin{aligned}
& \Omega_{f}= \pm \Omega_{0}\left(I_{f}+I_{0}\right) \sqrt{\left.\frac{I_{0}}{\Phi\left(I_{f}+I_{0}\right.}\right)^{-1}}, \\
& \vec{Q}= \pm \vec{Q}_{0} \Phi \sqrt{\frac{I_{0}}{\Phi\left(I_{f}+I_{0}\right)}-1}, \\
& g_{1,2}= \pm g_{0} \Phi \sqrt{\left.\frac{I_{0}}{\Phi\left(I_{f}+I_{0}\right.}\right)^{-1} \mp} \\
& \mp \sqrt{g_{0}^{2} \Phi^{2}\left(\frac{I_{0}}{\left.\Phi\left(I_{f}+I_{0}\right)^{-1}\right)}-1 \mp i \Omega_{0}\left(I_{f}+I_{0}\right) \sqrt{\left.\frac{I_{0}}{\Phi\left(I_{f}+I_{0}\right.}\right)^{-1}}\right.},
\end{aligned}
$$

here for $g_{1}$ and $g_{2}$ the signs "-" and "+" before "big" root must be correspondingly chosen, and other signs are simultaneously upper or lower in all values. $\Omega_{0}, Q_{0}, g_{0}$ are real constants, independent over $j$ and $\Phi$, which with-

$S Q O, 5(3), 2002$ out any restriction can be chosen positive. It is easy to verify that the asymptotes of dependences (41) coincide with (21), but (41) can be analyzed much simpler than (16-17). For this goal, let us rewrite (41) in the dimensionless variables:

$$
\begin{aligned}
& \Omega_{f}= \pm \Omega_{0}(j+1) \sqrt{\frac{j}{\Phi(j+1)}-1}, \\
& \vec{Q}= \pm \vec{Q}_{0} \Phi \sqrt{\frac{j}{\Phi(j+1)}-1}, \\
& g_{1,2}= \pm g_{0} \Phi \sqrt{\frac{j}{\Phi(j+1)}-1 \mp} \\
& \mp \sqrt{g_{0}^{2} \Phi^{2}\left(\frac{j}{\Phi(j+1)}-1\right)-1 \mp i \Omega_{0}(j+1) \sqrt{\frac{j}{\Phi(j+1)}-1}}, \\
& j=\frac{I_{0}}{I_{f}}, \quad 0<\Phi<1 .
\end{aligned}
$$

The dependences of $\Omega_{f} / \Omega_{0}, Q / Q_{0}$ and $g_{1,2} / g_{0}$ over $j$ are depicted in Fig.5. Notice, that

$$
\operatorname{Re}\left(g_{1}\right) \approx-\operatorname{Re}\left(g_{2}\right), \quad \operatorname{Im}\left(g_{1}\right)=-\operatorname{Im}\left(g_{2}\right) .
$$

Thus, under validity of (39) and in accordance with the Wiener-Hopf theorem, the boundary cycle with spectrum maximum (38-41) exists in the generalized nonlinear system. Its linearized master equation can be presented in the form (35a). Therefore, the modulated inner field (40) develops in the medium. The latter is due to EOeffect recording the phase dynamic holographic grating. In its turn, the laser pump wave will diffract on this oscillating grating.

Finally, in the case of essentially nonlinear restricted in transverse directions real systems, the following questions are desirable to answer: under which conditions the aforementioned boundary cycle can be stable and what kind of dynamic processes exists in these systems? Restricting itself by studying of transverse instabilities corresponding to the boundary conditions (35d), one can answer the questions having analyzed the dependence of transverse eigen vector imaginary part $q^{\prime \prime}{ }_{\perp}$ in (36) on frequency $\Omega$ (see Fig 6). Notice that the absolute dynamic instability threshold corresponds to $\operatorname{Im}(\Omega)=0$ in accordance with the subordination principle [19]. Thus, it is clear from (36) that $q_{\perp}(\Omega \rightarrow \pm \infty)=-\Lambda_{\perp} C / B, \quad q_{\perp}(\Omega=0)=-\Lambda_{\perp}$. Therefore, taking into account (36), the following conclusions can be done.

1) $C / B<0$. The aforementioned boundary cycle exists and can be stable if the frequency (35a) is less than the medium inertia one and the stationary point of the initial system is stable. Returning to the concrete system (1) and results (15-21) we can confirm that the periodic process found there is stable, because the stationary point is stable at $I_{0}<I_{C}$. This fact means that in the latter case the transverse periodical recharging waves must be observable in the aforementioned PR-materials. 
A.N. Morozovska et al.: Dynamics of photoinduced instability in ferroelectric...
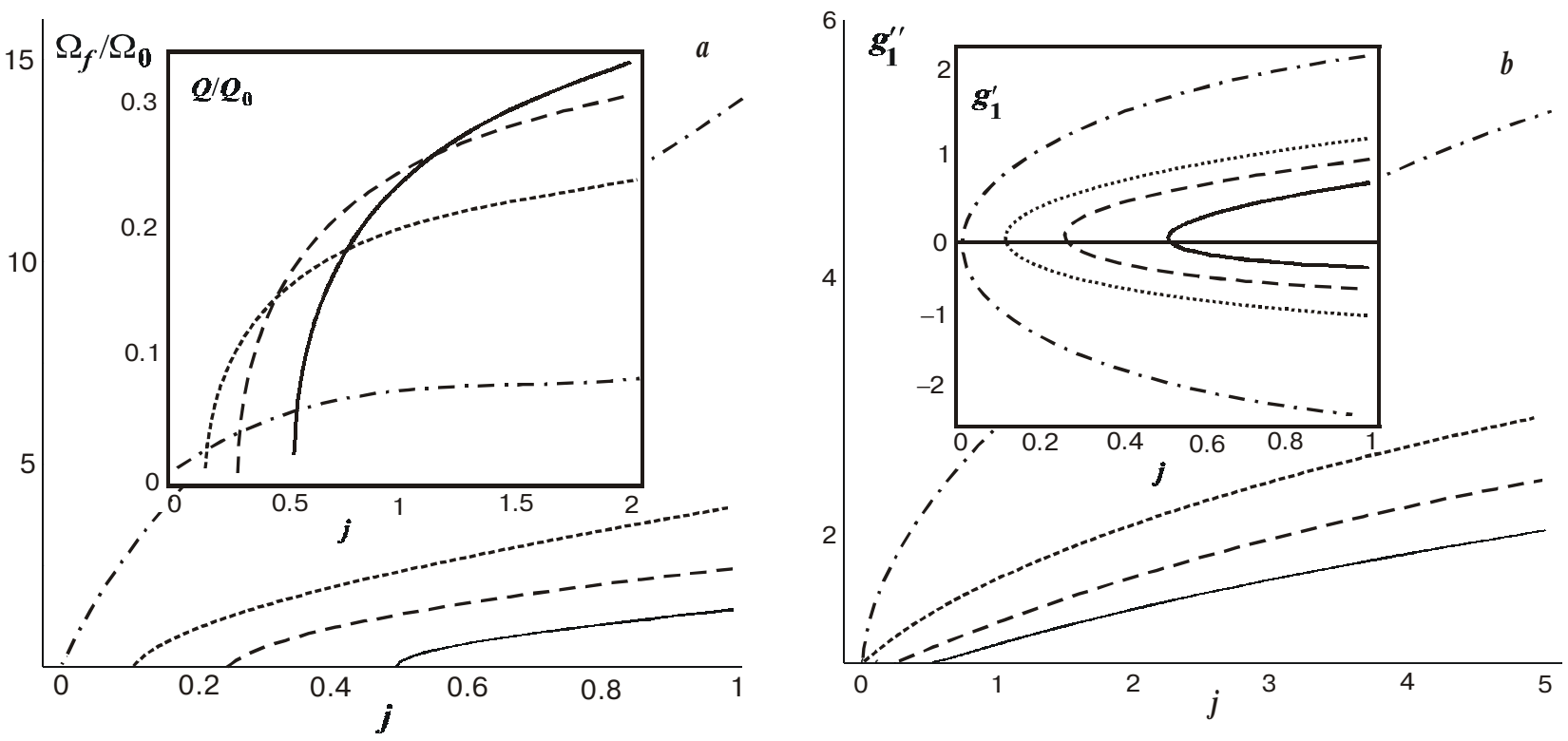

Fig.5. The dependences of $\Omega_{f} / \Omega_{0}, Q / Q_{0}(a)$ and complex coefficients $g_{1,2} / g_{0}(b)$ on $j$ at $\Omega_{0}=1, g_{0}=1$ and different $\Phi: \Phi=0.33$ (solid curves); 0.25 (dashed curves); 0.1 (dotted curves); 0.01 (dash-dotted curves).

2) If $0<C / B<1$, the dynamic transverse structures can appear under special initial conditions but with time the stationary field distribution will take place in the nonlinear system, i.e. the recharging waves are unstable.

3) If $C / B \geq 1$, the dynamic transverse instabilities with an infinite frequency are most energetically preferable for the nonlinear system. But the real frequency value is undoubtedly finite and restricted by the sluggishness of the PR-medium. That is the case when the stochastic behavior is most energetically preferable for the nonlinear system.

\section{Conclusion}

In this paper we have studied consecutively the influence of the recharging trap waves on the dynamics of the inner photoinduced fields in the uniaxial ferroelectric PRC under stationary laser irradiation. The characteristic features of the direct and inverse PILS have been predicted. The obtained results can be briefly formulated as following statements.

For the first time it has been shown that, due to influence of transverse photovoltaic current and the typical boundary conditions for all components of inner field, among all possible scenarios of transverse instabilities the boundary circle will be realized in the perfect crystal. Due to this phenomenon the periodical or quasi-periodical PILS appears in the real system, depending on the structure of growth non-homogeneities and the character of photoinduced fluctuations caused by them. The great attention has been paid to generalization of the results in the form of master equation and to description of the optical autowaves generation in ilmenites.

All the main theoretical results are in a good agreement with the available experimental data and describe the phenomena found in [6].

\section{References}

1. N.N. Rozanov, Hysteresis phenomena in distributed optical systems // JETP. 80(1), pp.96-108 (1981).

2. J. Takacs and L. Solymar, Subharmonics in $\mathrm{Bi}_{12} \mathrm{SiO}_{20}$ with an applied ac electric field // Opt.Lett. 17(2), pp.247-248 (1992).

3. D. Engin, S. Orlov, M. Segev, G.C. Valley, A. Yariv, Orderdisorder phase transition and critical slowing down in photorefractive self-oscillators // Phys.Rev. Lett. 74(10), pp.1743-1746 (1995).

4. I.V. Dzedolik, A.V. Volyar, The registration of optical vortices in refractive media // Proceedings of SPIE. 3904, pp. 8391 (1999).

5. Kh.S. Bagdasarov, V.B. Batoev, E.M. Uyukin, Non-stationary Isotropic Photoinduced Light Scattering in $\mathrm{LiTaO}_{3}: \mathrm{Cr} / /$ Quant.Elect. 13(10), pp.1962-1964 (1986).

6. V.V. Lemeshko, V.V. Obukhovskii, Autowaves of photoinduced light scattering // Pis'ma.Zh.Tekh.Fiz. 11(11), pp. 13891393 (1985).

7. O. Sandfuchs, J. Leonardy, F. Kaiser and M. Belic, Transverse instabilities in photorefractive counter propagating twowave mixing //Phys. Rev. A53(6), pp.4519-4524 (1996.)

8. T. Honda, P. Banerjee, Threshold for spontaneous pattern formation in reflection-grating-dominated photorefractive media with mirror feedback //Opt. Lett. 21(11), pp. 779-781 (1996).

9. B.I. Sturman, E. Sharmonina, M. Mann, K.H. Ringhofer, Space-charge waves in photorefractive ferroelectrics // J.Opt.Soc.Am. 12(9), pp. 1672-1650 (1995).

10. A.N.Morozovska, V.V. Obukhovskii, Autowave-type instability in photorefractive crystals // Proceedings of SPIE. 3904, pp. 209-214 (1999)

11. A.N. Morozovska, V.V.Obukhovskii, Dynamic halo-scattering in photorefractive crystals // Proceedings of SPIE. 4355, pp. 22-227 (2001).

12. A.S. Furman, Spontaneous increasing of recharging waves in crystals without inversion center under stationary illumination // Sol.St.Phys. 29(4), pp.1076-1085 (1985).

13. N.V. Kukhtarev, V.B. Markov, S.G. Odulov, M.M. Soskin, V.L. Vinetskii, Holographic Storage in Electrooptic crystals / / Ferroelectrics, 22, pp.949-960 (1979).

14. V.M. Fridkin, Photo-Ferroelectrics, M. Nauka, (1979) (in Rus).

$S Q O, 5(3), 2002$ 
A.N. Morozovska et al.: Dynamics of photoinduced instability in ferroelectric...

15. G.M. Guro, I.I. Ivanchik, N.F. Kovtonuk, C-domain crystal of barium titanate in shorted capacitor // Sol.St.Phys. 11(7), pp.1956-1964 (1969).

16. U.S. Kuzminov, Litium niobate and tantalate are the materials for nonlinear optics, M. Nauka (1975) (in Rus).

17. A. Yariv, P. Yeh, Optical waves in crystals, N.-Y. (1985).

18. M. Born, E. Wolf, The principles of optics, M.: Nauka (1973) (in Rus).

19. H. Haken, Synergetics. N.-Y.: Springer-Verlag. (1979).

20. O. Sandfuchs, J. Leonardy, F. Kaiser and M. Belic, Transverse instabilities in photorefractive counter propagating twowave mixing //Opt.Lett. 22(8), pp.498-500 (1997).

21. A.N.Morozovska, V.V. Obukhovskii, V.V. Lemeshko Dynamics of Photoinduced Instability in Ferroelectric Photorefractive Crystals.// OptElectr. In press (2002).
22. V.V. Obukhovskii, A.V. Stoyanov. A model of photogalvanic centers in crystals.// Ferroelectrics, 43(3/4), pp.137-142 (1982).

23. T.H. Yeom, S.H. Choh. Magnetic Resonance Investigations of $\mathrm{LiNbO}_{3}$ and $\mathrm{LiTaO}_{3}$ Single Crystals.// Journal of Korean Physical Society, 32, pp.S672-S674 (1998).

24. G.I. Malovichko, V.A. Grachev and S.N. Likin. EPR studies of axial and low symmetry centres $\mathrm{Gr}^{3+}, \mathrm{Fe}^{3+}, \mathrm{Mn}^{3+} / /$ Sov.Phys. Solid State, 28(4), pp.553-559 (1986).

25. T.H. Yeom, S.H. Lee, S.H. Choh, D. Choi. Electron Paramagnetic Resonance Study in $\mathrm{LiNbO}_{3}: \mathrm{Mg}: \mathrm{Fe}$ Crystal.// Journal of Korean Physical Society, 32, pp.S647-S649 (1998).

26. T.S. Yu, Introduction to diffraction, information processing and holography, MIT Press Cambridge, Massachusetts and London, England (1973). 